\title{
Collective diffusion in sheared colloidal suspensions
}

\author{
ALEXANDER M. LESHANSK Y ${ }^{1}$, \\ JEFFREY F. MORRIS ${ }^{2}$ AND JOHN F. BRADY \\ ${ }^{1}$ Department of Chemical Engineering, Technion-IIT, Haifa, 32000, Israel \\ ${ }^{2}$ Benjamin Levich Institute and Department of Chemical Engineering, City College of New York, \\ New York, NY 10031, USA \\ ${ }^{3}$ Division of Chemistry \& Chemical Engineering, California Institute of Technology, \\ Pasadena, CA 91125, USA
}

(Received 3 June 2007 and in revised form 18 October 2007)

Collective diffusivity in a suspension of rigid particles in steady linear viscous flows is evaluated by investigating the dynamics of the time correlation of long-wavelength density fluctuations. In the absence of hydrodynamic interactions between suspended particles in a dilute suspension of identical hard spheres, closed-form asymptotic expressions for the collective diffusivity are derived in the limits of low and high Péclet numbers, where the Péclet number $P e=\dot{\gamma} a^{2} / D_{0}$ with $\dot{\gamma}$ being the shear rate and $D_{0}=k_{B} T / 6 \pi \eta a$ is the Stokes-Einstein diffusion coefficient of an isolated sphere of radius $a$ in a fluid of viscosity $\eta$. The effect of hydrodynamic interactions is studied in the analytically tractable case of weakly sheared $(P e \ll 1)$ suspensions.

For strongly sheared suspensions, i.e. at high $P e$, in the absence of hydrodynamics the collective diffusivity $\boldsymbol{D}^{c}=6 \boldsymbol{D}_{\infty}^{s}$, where $\boldsymbol{D}_{\infty}^{s}$ is the long-time self-diffusivity and both scale as $\phi \dot{\gamma} a^{2}$, where $\phi$ is the particle volume fraction. For weakly sheared suspensions it is shown that the leading dependence of collective diffusivity on the imposed flow is proportional to $D_{0} \phi P e \hat{\boldsymbol{E}}$, where $\hat{\boldsymbol{E}}$ is the rate-of-strain tensor scaled by $\dot{\gamma}$, regardless of whether particles interact hydrodynamically. When hydrodynamic interactions are considered, however, correlations of hydrodynamic velocity fluctuations yield a weakly singular logarithmic dependence of the cross-gradient-diffusivity on $k$ at leading order as $a k \rightarrow 0$ with $k$ being the wavenumber of the density fluctuation. The diagonal components of the collective diffusivity tensor, both with and without hydrodynamic interactions, are of $O\left(\phi P e^{2}\right)$, quadratic in the imposed flow, and finite at $k=0$.

At moderate particle volume fractions, $0.10 \leqslant \phi \leqslant 0.35$, Brownian Dynamics (BD) numerical simulations in which there are no hydrodynamic interactions are performed and the transverse collective diffusivity in simple shear flow is determined via time evolution of the dynamic structure factor. The BD simulation results compare well with the derived asymptotic estimates. A comparison of the high- $P e \mathrm{BD}$ simulation results with available experimental data on collective diffusivity in non-Brownian sheared suspensions shows a good qualitative agreement, though hydrodynamic interactions prove to be important at moderate concentrations.

\section{Introduction}

Over a century after Einstein (1905) published his seminal paper on Brownian diffusion, the theory of particle diffusion in colloidal dispersions is still under development. Despite the tremendous progress in understanding of diffusion inspired 
by the Einstein work over the last 100 years, the theory of diffusion in non-equilibrium flowing suspension has developed only in the last 30 years. The major obstacle appears to be the non-trivial coupling between Brownian and hydrodynamic forces due to shearing motion. The influence of these factors is not simply additive, and shear-induced enhancement of particle diffusivity or, more generally, dispersion is anticipated as in the analogous problem of passive scalar transport (Taylor 1953; Novikov 1958; Elrick 1962; Batchelor 1979).

As is the case at equilibrium, in suspensions undergoing steady shear two distinct diffusive processes can be identified: the particle self-diffusivity, related to the rate of change of the variance in the position of a tagged particle, and the collective diffusivity (or gradient diffusivity) corresponding to a rate at which macroscopic spatial inhomogeneities in particle distribution relax. Also, as in a macroscopically quiescent suspension, the particles are expected to behave diffusively on two separate time scales characterized by short- and long-time diffusion coefficients (see Pusey 1991). The latter results from the hindering effect of the direct interactions (e.g. hydrodynamic or steric) between suspended particles as they move through the suspension. In sheared suspensions the spatial anisotropy of the imposed flow results in diffusion coefficients that are anisotropic and the variance in position of a particle may grow faster than linearly in time owing to the Taylor dispersion driven by the position-dependent imposed flow.

The early studies of diffusion in sheared systems (e.g. san Miguel \& Sancho 1979) used a transformation to a coordinate system co-moving with the shearing motion to remove the linear flow from the governing equations. In Frankel \& Brenner (1991), a generalized Taylor dispersion theory was developed to study transport of an isolated particle in linear flows. An appropriate transformation of the time coordinate was applied to remove the position-dependent advection of the bulk flow, but the complexity of the analysis may not allow it to be applied to study of the transport of interacting particles or collective diffusion.

At the extreme of strong shearing, there have been a number of studies of shearinduced or hydrodynamic diffusion (Eckstein, Bailey \& Shapiro 1977; Leighton \& Acrivos 1987; Breedveld et al. 1998). Trajectory analysis was applied in Acrivos et al. (1992) to calculate the self-diffusivity, scaling as $\phi \dot{\gamma} a^{2}$ in the flow direction, with $\dot{\gamma}$ being the shear rate and $a$ the sphere radius. The $O\left(\phi^{2}\right)$ self- and collectivediffusion coefficients in the velocity-gradient direction were determined by Wang, Mauri \& Acrivos (1996 and 1998, respectively) from trajectory analysis including three-particle interactions in a monolayer of spheres. $\dagger$ In da Cunha \& Hinch (1996), the diffusion coefficients were derived from the pair-trajectory calculations of spheres with slight surface roughness. A similar analysis was applied in Wang \& Mauri (1999) to calculate a collective-diffusion coefficient from analysis of pairwise interactions at $O(\phi)$. Nevertheless, applicability of trajectory analysis will probably remain limited to studies of diffusion in the absence of Brownian transport, because it is not clear how to introduce stochastic forces into the formulation.

An alternative approach was recently proposed by Mauri (2003) where homogenization techniques were applied to derive a constitutive relation for the coarse-grained

$\dagger$ The hydrodynamic encounter between two perfectly smooth spheres in Stokes flow does not lead to a net lateral displacement of either sphere due to the linearity and time-reversibility of the equations of motion. Thus, at the pair level a non-hydrodynamic microscopic mechanism (e.g. particle roughness, repulsive forces, Brownian forces, etc.) must be included to provide microscopic irreversibility that would lead to net displacement and diffusion upon many successive encounters. 
averaged volumetric flux of non-Brownian particles in an inhomogeneous and nonstationary viscous flow. Though the general constitutive relation for the collective diffusivity in terms of the velocity cross-correlation was derived, no comparison with existing experimental results or theoretical predictions have been performed. As in the trajectory technique discussed above, generalization of the method of Mauri (2003) to allow consideration of combined hydrodynamic and Brownian transport is missing.

Morris \& Brady (1996) presented a methodology stemming from the experimental technique of dynamic light scattering (Berne \& Pecora 1976) to calculate particle self-diffusivity in sheared suspensions, and applied it to the case of weak shearing (small Péclet number, $\mathrm{Pe}$ ). Although successful theories referring to dynamic light scattering had been proposed to study diffusion in quiescent colloidal dispersions of hydrodynamically interacting particles (Russel \& Glendinning 1981; Rallison \& Hinch 1986; Brady 1994), the generalization of the method by Morris \& Brady allowed investigation of particle transport in non-equilibrium sheared suspensions. The central idea of the equilibrium theories is based upon the relation between diffusivity (self- and collective) and the rate of temporal decay of the autocorrelation in number density fluctuations (decorrelation in scattered light is due to uncorrelated particle motions). This idea has been shown to have the same role in the theory of selfdiffusivity in sheared suspensions (Morris \& Brady 1996). Under shear the variance in particle position is not readily related to diffusion as in a quiescent suspensions owing to the Taylor dispersion driven by the position-dependent imposed flow, while the Fourier-transform method was shown to be useful in identifying particle selfdiffusivity by analogy with the treatment of passive scalar transport (see Batchelor 1979). The short-time self-diffusivity (the product of the average mobility of a particle and $k_{B} T$ ) and the long-time self-diffusivity (due to the correlation of the flux of the tagged particles with the microstructural perturbation caused by their motion) were determined at $O(\phi)$; note that the microstructure associated with the short-time self-diffusivity is that of the steadily sheared suspension at the shear rate of interest. In a later paper, Brady \& Morris (1997) demonstrated that the same methodology is applicable to study the effect of strong shear on self-diffusivity and to predict the $O(\phi)$ coefficient of the $\dot{\gamma} a^{2}$ self-diffusivity in a general linear flow. The obvious advantage of the Fourier-transform method is that it places the analysis of quiescent and flowing suspensions on the same footing. It was suggested in Morris \& Brady (1996) that the approach can be applied to calculation of the collective diffusivity in sheared colloidal suspensions, as done for the quiescent suspension by Rallison \& Hinch (1986), but no such study has been attempted prior to the present work.

Although self-diffusion coefficients can be calculated using appropriate kinematic descriptions, such as the mean-square displacement or velocity autocorrelation function of a particle, and these apply even in highly non-equilibrium systems (Marchioro \& Acrivos 2001; Drazer et al. 2002; Sierou \& Brady 2004), evaluation of the gradient diffusivity is less obvious. For macroscopically quiescent suspensions there is a clear physical description of the short-time collective diffusivity through the wave-vector-dependent diffusion coefficient $D(k)$, which depends on both structural and hydrodynamic effects (Russel \& Glendinning 1981; Pusey 1991; Segré, Behrend \& Pusey 1995),

$$
\frac{\mathscr{D}(k)}{D_{0}}=\frac{\mathscr{H}(k)}{S(k)},
$$

where $k$ is the magnitude of the wave-vector of the density fluctuation, $\mathscr{H}(k)$ represents purely hydrodynamic effects, and structural effects are contained in the static structure 
factor $S(k)$. When $a k \ll 1$, we are in the long-wavelength limit where the scale of the density variation is large relative to the particle size. The relaxation of longwavelength density fluctuations yields a particle flux which is the same as if a small constant density gradient persisted everywhere (Rallison \& Hinch 1986). Therefore, as $k a \rightarrow 0$, one expects that $\mathscr{D}(k)$ will asymptote to the constant collective or downgradient diffusivity, $D^{c}$. Also in the limit of vanishing $k$, the magnitude of the density fluctuations of a system, as characterized by the static structure factor $S(0)$, is related to its osmotic or isothermal compressibility $S(0)=k_{B} T(\partial n / \partial \Pi)_{T}$ (Berne \& Pecora 1976; Pusey 1991). Therefore, $S(0)^{-1}$ can be interpreted as a thermodynamic force associated with the density gradient which drives the diffusion. The hydrodynamic factor, $\mathscr{H}(k)$, can be expressed through an integral over the velocity cross-correlation (Pusey 1991; Segré et al. 1995). For a dilute colloidal suspension of hard spheres, $S(0) \sim 1-8 \phi$ due to excluded volume effects, while the collective mobility is given by the mean sedimentation velocity, relative to zero volume-flux axes $\mathscr{H}(0) \sim(6 \pi \eta a)^{-1}(1-6.55 \phi)$, leading to the classical result $D^{c}=k_{B} T \mathscr{H}(0) / S(0) \sim D_{0}(1+1.45 \phi)$ (Batchelor 1976); more accurate hydrodynamic data yield a slightly different value of $1.47 \phi$ for the last term in parentheses (Rallison \& Hinch 1986).

In Marchioro \& Acrivos (2001), a novel numerical scheme for calculating the gradient diffusivity in non-Brownian suspensions, which should also be applicable to the case with Brownian motion, using Stokesian Dynamics simulations has been proposed. The essence of the method is that the suspension microstructure relaxes at a rate proportional to the gradient-diffusion coefficient. Thus, if an initial microstructure is sufficiently different from that of a suspension achieved after extended steady shearing, probing the rate of relaxation from the initial toward the steady microstructure provides a means of determining the gradient diffusivity.

A different approach was recently proposed in Leshansky \& Brady (2005), where it was demonstrated that representation (1.1) holds for the tensorial wave-vectordependent coefficient $\mathscr{D}$ of suspensions in arbitrary steady linear flow. Again the method was applied to describe the relaxation of the long-wavelength density fluctuations, $a k \rightarrow 0$. The work showed that the tensorial hydrodynamic factor $\mathscr{H}$ can be expressed through the averaged integral of the velocity cross-correlation function and $S(0)$ can be interpreted as that corresponding to the non-equilibrium shear-induced suspension microstructure. This approach is conceptually different from that of Marchioro \& Acrivos (2001) as it concerns the decay of the fluctuations in number density rather than relaxation of the macroscopic density itself. For the selfdiffusivity this approach yields a correct description of the self-diffusivity in terms of the integral over the velocity autocorrelation derived earlier from purely kinematic arguments by Marchioro \& Acrivos (2001) and Sierou \& Brady (2004). The results for the shear-induced collective-diffusion coefficients (in the absence of Brownian transport) in the velocity-gradient and vorticity directions obtained in Leshansky \& Brady from accelerated Stokesian Dynamics simulations (Sierou \& Brady 2001) show very good agreement with available experimental data. The derivation is independent of the details of the microscale dynamics and thus should hold quite generally.

The present paper is a natural continuation of the work in Morris \& Brady (1996), Brady \& Morris (1997), and Leshansky \& Brady (2005). We address the problem of calculating the collective diffusivity in a suspension of hard spheres in an arbitrary steady linear flow at small Reynolds number. The light-scattering-based method of Morris \& Brady is applied to investigation of the rate of decay of the density fluctuations. 
The paper is organized as follows. In $\S 2$ we show how the collective diffusivity can be identified via the dynamic structure factor approach and derive the closed-form asymptotic expression for the tensorial diffusion coefficient in the dilute limit for weakly and strongly sheared suspensions. In $\S 3$ we briefly describe the details of the Brownian Dynamics simulations and present results for the transverse diffusivities along both principal directions of a simple shear flow (velocity gradient and vorticity). In $\S 4$ a brief summary and concluding remarks are provided. The Appendix concerns the long-wavelength microstructural deformation of a suspension in simple shear flow in terms of the non-equilibrium static structure factor $S(k=0)$.

\section{Theoretical development}

\subsection{Dynamic structure factor approach}

To understand how we can define the particle diffusivity in linear flow, consider the following conservation equation for the local particle number density, $n(\boldsymbol{x}, t)$ :

$$
\frac{\partial n}{\partial t}+\dot{\boldsymbol{\Gamma}} \cdot \boldsymbol{x} \cdot \nabla n+\boldsymbol{U} \cdot \nabla n=-\nabla \cdot \boldsymbol{j}
$$

where $\dot{\boldsymbol{\Gamma}}$ is the constant velocity-gradient tensor, $\boldsymbol{U}$ is the bulk average velocity measured at an arbitrary field point, $\boldsymbol{x}_{0}$, from which the bulk shear velocity is referenced and $\boldsymbol{j}$ is the diffusive flux of particles. Neglecting memory effects for time scales over which the diffusion is stationary (either short or long time scale), the flux should be expressible as a generalized Fick's law:

$$
\boldsymbol{j}=-\int \mathscr{D}\left(\boldsymbol{x}-\boldsymbol{x}^{\prime}\right) \cdot \nabla n\left(\boldsymbol{x}^{\prime}, t\right) \mathrm{d} \boldsymbol{x}^{\prime},
$$

where the non-local kernel is identified as the effective diffusivity with dimensions $(L T)^{-1}$. With the definition of the Fourier transform $(\mathscr{F})$ pair

$$
\widehat{\boldsymbol{h}}(\boldsymbol{k})=\int \boldsymbol{h}(\boldsymbol{x}) \mathrm{e}^{-\mathrm{i} \boldsymbol{k} \cdot \boldsymbol{x}} \mathrm{d} \boldsymbol{x}, \quad \boldsymbol{h}(\boldsymbol{x})=(2 \pi)^{-3} \int \widehat{\boldsymbol{h}}(\boldsymbol{k}) \mathrm{e}^{\mathrm{i} \boldsymbol{k} \cdot \boldsymbol{x}} \mathrm{d} \boldsymbol{k},
$$

where $\boldsymbol{k}$ is the wave-vector in Fourier space, and using the identity

$$
\int \boldsymbol{G}(\boldsymbol{x}-\boldsymbol{y}) \cdot \boldsymbol{W}(\boldsymbol{y}) \mathrm{d} \boldsymbol{y}=(2 \pi)^{-3} \int \widehat{\boldsymbol{W}}(\boldsymbol{k}) \cdot \widehat{\boldsymbol{G}}(\boldsymbol{k}) \mathrm{e}^{\mathrm{i} \boldsymbol{k} \cdot \boldsymbol{x}} \mathrm{d} \boldsymbol{k},
$$

the expression for flux (2.2) can be rewritten as

$$
\boldsymbol{j}=-(2 \pi)^{-3} \int \widehat{\mathscr{D}}\left(\boldsymbol{k}^{\prime}\right) \cdot \mathrm{i} \boldsymbol{k}^{\prime} \widehat{n}\left(\boldsymbol{k}^{\prime}, t\right) \mathrm{e}^{\mathrm{i} \boldsymbol{k}^{\prime} \cdot \boldsymbol{x}} \mathrm{d} \boldsymbol{k}^{\prime} .
$$

Applying the Fourier transform to (2.3) and using the identity $\mathscr{F}\left\{\mathrm{e}^{\mathrm{i} x \cdot\left(k^{\prime}-k\right)}\right\}=$ $(2 \pi)^{3} \delta\left(\boldsymbol{k}^{\prime}-\boldsymbol{k}\right)$, we arrive at the Fourier-space expression for $\boldsymbol{j}$ :

$$
\widehat{\boldsymbol{j}}=-\widehat{\mathscr{D}}(\boldsymbol{k}) \cdot \mathrm{i} \boldsymbol{k} \widehat{n}(\boldsymbol{k}, t),
$$

where the $\boldsymbol{k}$-dependent diffusivity $\widehat{\mathscr{D}}(\boldsymbol{k})$ has the dimensions $L^{2} / T$. The Fourier transform applied to the left-hand side of (2.1) combined with (2.4) converts (2.1) to

$$
\frac{\partial \widehat{n}}{\partial t}-\boldsymbol{k} \cdot \dot{\boldsymbol{\Gamma}} \cdot \nabla_{k} \widehat{n}-\mathrm{i} \boldsymbol{k} \cdot \boldsymbol{U} \widehat{n}=-\boldsymbol{k} \cdot \widehat{\mathscr{D}}(\boldsymbol{k}) \cdot \boldsymbol{k} \widehat{n} .
$$

This equation is analogous to that which describes the evolution of a passive scalar field in a linear flow, in which case there would be a constant isotropic diffusivity 
$\widehat{\mathscr{D}}=D I$. Note that the spreading or dispersion of the passive scalar due to the shear flow, characterized by the second moment $\int \boldsymbol{x} \boldsymbol{x} n(\boldsymbol{x}, t) \mathrm{d} \boldsymbol{x}$, may grow faster than linearly in time. This is the well-known Taylor dispersion (Taylor 1953; Novikov $1958)$ and is accounted for by the $\boldsymbol{k} \cdot \dot{\boldsymbol{\Gamma}} \cdot \nabla_{k} \widehat{n}$ term on the left-hand side of (2.5). The general solution of (2.5) for an arbitrary linear flow can be found for a constant $\boldsymbol{k}$-independent tensorial coefficient $\widehat{\mathscr{D}}$ (Batchelor 1979).

In a steadily sheared suspension the average density fluctuation is zero and therefore we consider the two-point time autocorrelation of the local particle density, $F(\boldsymbol{k}, t)=\left\langle\widehat{n}(\boldsymbol{k}, t) \widehat{n}^{*}(\boldsymbol{k}, 0)\right\rangle$, where * indicates a complex conjugate and angle brackets denote an appropriate statistical averaging (either ensemble or time averaging). Multiplying both sides of $(2.5)$ by $\widehat{n}^{*}(\boldsymbol{k}, 0)$ the evolution equation for $F$ becomes

$$
\frac{\partial F}{\partial t}-\boldsymbol{k} \cdot \dot{\boldsymbol{\Gamma}} \cdot \nabla_{k} F-\mathrm{i} \boldsymbol{k} \cdot \boldsymbol{U} F+\left\langle\widehat{n} \boldsymbol{k} \cdot \dot{\boldsymbol{\Gamma}} \cdot \nabla_{k} \widehat{n}^{*}\right\rangle=-\boldsymbol{k} \cdot \mathscr{D}(\boldsymbol{k}) \cdot \boldsymbol{k} F,
$$

where we have removed the hat over $\mathscr{D}$ for simplicity. The above equations for $\widehat{n}$ and $F$ have the expected form for a diffusive process in a linear flow, and can be used to identify the proper expressions for the particle diffusivities either from statistical mechanical theory or from dynamic simulations of multiparticle systems. Note that the last term on the left-hand side of (2.6) may lead to a modification of the particle dispersion in comparison with the dispersion of a passive scalar as described by (2.5), where this term is missing. In Morris \& Brady (1996), the working hypothesis was that the relaxation of the scalar field $F$ is governed by (2.5) simply by analogy and, therefore, this term was omitted. (Retaining the last term on the left-hand side of (2.6) does not alter the outcome of the analogous analysis for the self-diffusivity in Morris \& Brady (1996) as the diffusive variation of $F$ is $O\left(k^{2}\right)$, while linear flow causes variation which is independent of $k$.)

As in macroscopically quiescent suspensions (e.g. Russel \& Glendinning 1981) the limiting value of $\mathscr{D}$ as $k \rightarrow 0$ is identified as the collective-diffusion coefficient $\boldsymbol{D}^{c}$.

To make analytical progress, the particle number density at any point in the suspension $\boldsymbol{x}$ is defined in terms of distributions as

$$
n(\boldsymbol{x}, t)=\sum_{\alpha=1}^{N} \delta\left(\boldsymbol{x}-\boldsymbol{x}_{\alpha}(t)\right),
$$

where $\delta$ is the Dirac delta function, and $\boldsymbol{x}_{\alpha}(t)$ is the position of particle $\alpha$ at time $t$. The spatial Fourier transform of $n(\boldsymbol{x})$ is

$$
\hat{n}(\boldsymbol{k}, t)=\int \mathrm{e}^{\mathrm{i} k \cdot \boldsymbol{x}} \sum_{\alpha=1}^{N} \delta\left(\boldsymbol{x}-\boldsymbol{x}_{\alpha}(t)\right) \mathrm{d} \boldsymbol{x}=\sum_{\alpha=1}^{N} \mathrm{e}^{\mathrm{i} \boldsymbol{k} \cdot \boldsymbol{x}_{\alpha}} .
$$

The density autocorrelation function, known as the dynamic structure factor or as the intermediate scattering function (primarily in colloidal and polymer literature), is given by

$$
F(\boldsymbol{k}, t)=\frac{1}{N}\left\langle\hat{n}(\boldsymbol{k}, t) \hat{n}^{*}(\boldsymbol{k}, 0)\right\rangle=\frac{1}{N}\left\langle\sum_{\alpha, \beta}^{N} \mathrm{e}^{\mathrm{i} k \cdot\left(\boldsymbol{x}_{\alpha}(t)-\boldsymbol{x}_{\beta}(0)\right)}\right\rangle,
$$

where the angular brackets \langle\rangle denote an ensemble average over the joint probability $P_{N}\left(\boldsymbol{x}^{N}(t), t ; \boldsymbol{x}^{N}(0), 0\right)$ of particles being at $\boldsymbol{x}^{N}(0)$ at time 0 and at $\boldsymbol{x}^{N}(t)$ at time $t$. The constant background density level of $n$ in (2.9) is irrelevant and thus $F$ describes the 
autocorrelation of density fluctuations (Berne \& Pecora 1976). Integrating over the joint probability density (2.9) yields, for statistically indistinguishable particles,

$$
\begin{aligned}
F(\boldsymbol{k}, t)= & \frac{1}{N} \frac{1}{N !} \int \sum_{\alpha, \beta}^{N} \mathrm{e}^{\mathrm{i} \boldsymbol{k} \cdot\left(x_{\alpha}(t)-x_{\beta}(0)\right)} P_{N}\left(\boldsymbol{x}^{N}(t), \boldsymbol{x}^{N}(0)\right) \mathrm{d} \boldsymbol{x}^{N} \mathrm{~d} \boldsymbol{x}^{N}(0) \\
= & \frac{1}{N !} \int\left(\mathrm{e}^{\mathrm{i} k \cdot\left(x_{1}(t)-x_{1}(0)\right)}+(N-1) \mathrm{e}^{\mathrm{i} \boldsymbol{k} \cdot\left(x_{2}(t)-\boldsymbol{x}_{1}(0)\right)}\right. \\
& \times P_{N} \mathrm{~d} \boldsymbol{x}^{N}(t) \mathrm{d} \boldsymbol{x}^{N}(0) \\
= & F_{S}(\boldsymbol{k}, t)+\frac{N-1}{N !} \iint \mathrm{e}^{\mathrm{i} \boldsymbol{k} \cdot\left(x_{2}-x_{1}(0)\right)} P_{N} \mathrm{~d} \boldsymbol{x}^{N}(t) \mathrm{d} \boldsymbol{x}^{N}(0),
\end{aligned}
$$

where we have introduced the self-dynamic structure factor (or self-intermediate scattering function) $F_{s}(\boldsymbol{k}, t)$.

Following Rallison \& Hinch (1986), we further define

$$
\hat{P}_{N}\left(\boldsymbol{x}^{N}, t ; \boldsymbol{k}\right)=\int P_{N}\left(\boldsymbol{x}^{N} \mid \boldsymbol{x}^{N}(0)\right) P_{N}^{0}\left(\boldsymbol{x}^{N}(0)\right) \mathrm{e}^{-\mathrm{i} \boldsymbol{k} \cdot \boldsymbol{x}_{1}(0)} \mathrm{d} \boldsymbol{x}^{N}(0),
$$

where the transitional probability $P_{N}\left(\boldsymbol{x}^{N} \mid \boldsymbol{x}^{N}(0)\right)$ is governed by a Smoluchowski equation (Morris \& Brady 1996)

$$
\frac{\partial P_{N}}{\partial t}+\sum_{\alpha=1}^{N} \nabla_{\alpha} \cdot j_{\alpha}=0,
$$

with initial value $\delta\left(\boldsymbol{x}^{N}-\boldsymbol{x}^{N}(0)\right)$ at $t=0$. In (2.12), $\boldsymbol{j}_{\alpha}$ is the probability flux associated with particle $\alpha$, given by

$$
\boldsymbol{j}_{\alpha}=\boldsymbol{U}_{\alpha} P_{N}-\sum_{\beta=1}^{N} \boldsymbol{D}_{\alpha \beta} P_{N} \cdot \nabla_{\beta}\left(\ln P_{N}+V\right),
$$

where $\boldsymbol{D}_{\alpha \beta}=k_{B} T \boldsymbol{M}_{\alpha \beta}$ with $\boldsymbol{M}_{\alpha \beta}$ the hydrodynamic mobility of particle $\alpha$ due to a force on particle $\beta, V\left(\boldsymbol{r}^{N}\right)$ is the interparticle potential (independent of absolute position) and $\boldsymbol{U}_{\alpha}$ is the hydrodynamic velocity that particle $\alpha$ would have due to external forces and bulk imposed flow, in the absence of Brownian motion and an interparticle potential.

Integrating in (2.10) over the initial coordinates $x^{N}(0)$ using (2.11) yields the expressions for $F$ and $F_{s}$, respectively,

$$
F=\frac{1}{N !} \int\left[1+(N-1) \mathrm{e}^{\mathrm{i} k \cdot r_{2}}\right] \hat{P}_{N} \mathrm{e}^{\mathrm{i} k \cdot x_{1}} \mathrm{~d} \boldsymbol{x}_{1} d \boldsymbol{r}^{N}, \quad F_{s}=\frac{1}{N !} \int \hat{P}_{N} \mathrm{e}^{\mathrm{i} k \cdot x_{1}} \mathrm{~d} \boldsymbol{x}_{1} \mathrm{~d} \boldsymbol{r}^{N},
$$

where the new coordinates

$$
\boldsymbol{x}_{1} \quad \text { and } \quad \boldsymbol{r}^{N} \equiv\left(\boldsymbol{r}_{2}, \ldots, \boldsymbol{r}_{N}\right)
$$

have been introduced where $\boldsymbol{x}_{1}=\boldsymbol{x}_{1}^{\prime}$ and $\boldsymbol{r}_{\alpha}=\boldsymbol{x}_{\alpha}^{\prime}-\boldsymbol{x}_{1}^{\prime}, \alpha \geqslant 2$, with the original variables denoted by primes. The formulation for the collective diffusivity can be expressed in terms of the configuration-dependent perturbation function $f_{N}\left(\boldsymbol{r}^{N}, t ; \boldsymbol{k}\right)$,

$$
\hat{P}_{N} \equiv \hat{P}_{1} P_{N-1 \mid 1}^{0}\left[1+f_{N}\right] \equiv \hat{P}_{1} Q,
$$

where

$$
\hat{P}_{1}\left(\boldsymbol{x}_{1}, t ; \boldsymbol{k}\right)=\frac{1}{(N-1) !} \int \hat{P}_{N} \mathrm{~d} \boldsymbol{r}^{N}
$$


is the probability density for particle 1 chosen at random (Morris \& Brady 1996) and the function $P_{N-1 \mid 1}^{0}\left(\boldsymbol{r}^{N}\right)$ is the steady non-equilibrium conditional probability of finding $N-1$ particles given that there is particle 1 at $\boldsymbol{x}_{1}$.

Using the Smoluchowski equation (2.12), governing the conservation of $\hat{P}_{N}\left(\boldsymbol{x}_{1}, \boldsymbol{r}^{N} ; \boldsymbol{k}\right)$, and integrating by parts, we arrive at

$$
\begin{aligned}
\dot{F} & =\frac{1}{N !} \int\left[1+(N-1) \mathrm{e}^{\mathrm{i} k \cdot \boldsymbol{r}_{2}}\right] \mathrm{e}^{\mathrm{i} k \cdot \boldsymbol{x}_{1}} \frac{\partial \hat{P}_{N}}{\partial t} \mathrm{~d} \boldsymbol{x}_{1} d \boldsymbol{r}^{N} \\
& =\mathrm{i} \boldsymbol{k} \cdot \frac{1}{N !} \int\left[\hat{\boldsymbol{j}}_{1}+(N-1) \hat{\boldsymbol{j}}_{2} \mathrm{e}^{\mathrm{i} \boldsymbol{k} \cdot \boldsymbol{r}_{2}}\right] \mathrm{e}^{\mathrm{i} \boldsymbol{k} \cdot \boldsymbol{x}_{1}} \mathrm{~d} \boldsymbol{x}_{1} \mathrm{~d} \boldsymbol{r}^{N},
\end{aligned}
$$

where $\hat{\boldsymbol{j}}_{\alpha}$ is the probability flux associated with particle $\alpha$ given by (2.13), but now in the new coordinates $\left(\boldsymbol{x}_{1}, \boldsymbol{r}^{N}\right)$, instantaneously centred at $\boldsymbol{x}_{1}$,

$$
\hat{\boldsymbol{j}}_{\alpha}=\boldsymbol{U}_{\alpha} \hat{P}_{N}-\boldsymbol{D}_{\alpha 1} \cdot \nabla_{1} \hat{P}_{N}-\sum_{\beta=2}^{N}\left(\boldsymbol{D}_{\alpha \beta}-\boldsymbol{D}_{\alpha 1}\right) \hat{P}_{N} \cdot \nabla_{\beta}\left(\ln \hat{P}_{N}+V\right), \quad 1 \leqslant \alpha \leqslant N .
$$

For an arbitrary linear flow $\boldsymbol{U}_{\alpha}$ may be written as

$$
\boldsymbol{U}_{\alpha}=\dot{\boldsymbol{\Gamma}} \cdot\left(\boldsymbol{r}_{\alpha}+\boldsymbol{x}_{1}\right)+\boldsymbol{U}^{*}+\boldsymbol{U}_{\alpha}^{\prime}\left(\boldsymbol{r}^{N}\right), \quad \alpha \geqslant 2,
$$

where $\dot{\boldsymbol{\Gamma}}$ is the constant traceless velocity-gradient tensor, $\boldsymbol{U}^{*}=\boldsymbol{U}^{\infty}\left(\boldsymbol{x}_{0}\right)-\dot{\boldsymbol{\Gamma}} \cdot \boldsymbol{x}_{0}$ with $\boldsymbol{U}^{\infty}$ being the bulk average velocity measured at an arbitrary field point, $\boldsymbol{x}_{0}$, and $\boldsymbol{U}_{\alpha}^{\prime}$ is the configuration-dependent velocity fluctuation. Equation (2.18) holds for particle 1 upon substitution $\boldsymbol{r}_{1}=0$. Substituting (2.17) into (2.16) and taking into account that $F_{s}=N^{-1} \int \hat{P}_{1} \mathrm{e}^{\mathrm{i} k \cdot x_{1}} \mathrm{~d} \boldsymbol{x}_{1}$ we integrate out $\boldsymbol{x}_{1}$ to obtain

$$
\begin{aligned}
\dot{F}= & \boldsymbol{k} \cdot \dot{\boldsymbol{\Gamma}} \cdot \nabla_{k} F-\frac{1}{N}\left\langle\widehat{n} \boldsymbol{k} \cdot \dot{\boldsymbol{\Gamma}} \cdot \nabla_{k} \widehat{n}^{*}\right\rangle+\mathrm{i} \boldsymbol{k} \cdot \boldsymbol{U}^{*} F \\
& -F_{s} \boldsymbol{k} \boldsymbol{k}: \frac{1}{(N-1) !} \int\left[\boldsymbol{D}_{11}+(N-1) \boldsymbol{D}_{21} \mathrm{e}^{\mathrm{i} k \cdot \boldsymbol{r}_{2}}\right] Q \mathrm{~d} \boldsymbol{r}^{N} \\
& +F_{s} \mathrm{i} \boldsymbol{k} \cdot \frac{1}{(N-1) !} \int\left[\boldsymbol{U}_{1}^{\prime}+(N-1) \boldsymbol{U}_{2}^{\prime} \mathrm{e}^{\mathrm{i} \boldsymbol{k} \cdot \boldsymbol{r}_{2}}\right] Q \mathrm{~d} \boldsymbol{r}^{N} \\
& -F_{s} \mathrm{i} \boldsymbol{k} \cdot \frac{1}{(N-2) !} \int\left[\boldsymbol{D}_{12}-\boldsymbol{D}_{11}+\left(\boldsymbol{D}_{22}-\boldsymbol{D}_{21}\right) \mathrm{e}^{\mathrm{i} k \cdot \boldsymbol{r}_{2}}\right] Q \cdot \nabla_{2}[\ln Q+V] \mathrm{d} \boldsymbol{r}^{N} \\
& -F_{s} \mathrm{i} \boldsymbol{k} \cdot \frac{1}{(N-3) !} \int\left(\boldsymbol{D}_{23}-\boldsymbol{D}_{21}\right) \mathrm{e}^{\mathrm{i} \boldsymbol{k} \cdot \boldsymbol{r}_{2}} Q \cdot \nabla_{3}[\ln Q+V] \mathrm{d} \boldsymbol{r}^{N} .
\end{aligned}
$$

The first two terms on the right-hand side of (2.19) result from the undisturbed linear velocity in (2.18):

$$
\begin{aligned}
\mathrm{i} \boldsymbol{k} \cdot \frac{1}{N !} \int\left[\dot{\boldsymbol{\Gamma}} \cdot \boldsymbol{x}_{1}+(N-1) \dot{\boldsymbol{\Gamma}} \cdot\left(\boldsymbol{r}_{2}+\boldsymbol{x}_{1}\right) \mathrm{e}^{\mathrm{i} \boldsymbol{k} \cdot \boldsymbol{r}_{2}}\right] \mathrm{e}^{\mathrm{i} \boldsymbol{k} \cdot \boldsymbol{x}_{1}} \hat{P}_{N} \mathrm{~d} \boldsymbol{x}_{1} \mathrm{~d} \boldsymbol{r}^{N} \\
\quad=\frac{1}{N}\left\langle\widehat{n}^{*}(\boldsymbol{k}, 0) \boldsymbol{k} \cdot \dot{\boldsymbol{\Gamma}} \cdot \nabla_{k} \widehat{n}(\boldsymbol{k}, t)\right\rangle=\boldsymbol{k} \cdot \dot{\boldsymbol{\Gamma}} \cdot \nabla_{k} F-\frac{1}{N}\left\langle\widehat{n} \boldsymbol{k} \cdot \dot{\boldsymbol{\Gamma}} \cdot \nabla_{k} \widehat{n}^{*}\right\rangle .
\end{aligned}
$$

We can simplify (2.19) further by noting that the total velocity disturbance must vanish, i.e.

$$
\int\left[\boldsymbol{U}_{1}^{\prime}+(N-1) \boldsymbol{U}_{2}^{\prime}\right] Q \mathrm{~d} \boldsymbol{r}^{N}=0
$$


so that the second integral on the right-hand side in (2.19) can be rewritten

$$
F_{s} \mathrm{i} \boldsymbol{k} \cdot \frac{1}{(N-2) !} \int \boldsymbol{U}_{2}^{\prime}\left(\mathrm{e}^{\mathrm{i} k \cdot \boldsymbol{r}_{2}}-1\right) Q \mathrm{~d} \boldsymbol{r}^{N} .
$$

Next we note that the particle mobility $\boldsymbol{D}_{11}$ is given by the isolated-particle value $D_{0} \boldsymbol{I}$ plus the contribution from $N-1$ other particlest, i.e. $\boldsymbol{D}_{11}=D_{0} \boldsymbol{I}+(N-1) \boldsymbol{D}_{11}^{\prime}$. Further, for identical particles

$$
\boldsymbol{D}_{12}-\boldsymbol{D}_{11}+\boldsymbol{D}_{22}-\boldsymbol{D}_{21} \equiv 0
$$

Thus we have

$$
\begin{aligned}
\dot{F}= & \boldsymbol{k} \cdot \dot{\boldsymbol{\Gamma}} \cdot \nabla_{k} F-\frac{1}{N}\left\langle\widehat{n} \boldsymbol{k} \cdot \dot{\boldsymbol{\Gamma}} \cdot \nabla_{k} \widehat{n}^{*}\right\rangle+\mathrm{i} \boldsymbol{k} \cdot \boldsymbol{U}^{*} F-F_{s} D k^{2} \\
& -F_{s} \boldsymbol{k} \boldsymbol{k}: \frac{1}{(N-2) !} \int\left(\boldsymbol{D}_{11}^{\prime}+\boldsymbol{D}_{21} \mathrm{e}^{\mathrm{i} k \cdot \boldsymbol{r}_{2}}\right) Q \mathrm{~d} \boldsymbol{r}^{N} \\
& +F_{s} \mathrm{i} \boldsymbol{k} \cdot \frac{1}{(N-2) !} \int \boldsymbol{U}_{2}^{\prime}\left(\mathrm{e}^{\mathrm{i} k \cdot \boldsymbol{r}_{2}}-1\right) Q \mathrm{~d} \boldsymbol{r}^{N} \\
& -F_{s} \mathrm{i} \boldsymbol{k} \cdot \frac{1}{(N-2) !} \int\left[\left(\boldsymbol{D}_{22}-\boldsymbol{D}_{21}\right)\left(\mathrm{e}^{\mathrm{i} \boldsymbol{k} \cdot \boldsymbol{r}_{2}}-1\right)\right] Q \cdot \nabla_{2}[\ln Q+V] \mathrm{d} \boldsymbol{r}^{N} \\
& -F_{s} \mathrm{i} \boldsymbol{k} \cdot \frac{1}{(N-3) !} \int\left(\boldsymbol{D}_{23}-\boldsymbol{D}_{21}\right) \mathrm{e}^{\mathrm{i} k \cdot \boldsymbol{r}_{2}} Q \cdot \nabla_{3}[\ln Q+V] \mathrm{d} \boldsymbol{r}^{N} .
\end{aligned}
$$

Substituting (2.15) into $F$ as given by (2.14) and integrating over $x_{1}$, it appears that $F$ is linear in $F_{s}$ :

$$
F(\boldsymbol{k}, t)=F_{s} \frac{1}{(N-1) !} \int\left[1+(N-1) \mathrm{e}^{\mathrm{i} \boldsymbol{k} \cdot \boldsymbol{r}_{2}}\right] Q \mathrm{~d} \boldsymbol{r}^{N} .
$$

Here we have made use of the fact that $F_{s}(0, t)=1$, so that $F_{s}(\boldsymbol{k}, t) \delta(\boldsymbol{k})=\delta(\boldsymbol{k})$. Since the average perturbation must be zero,

$$
\int P_{(N-1) \mid 1}^{0} f_{N} \mathrm{~d} \boldsymbol{x}^{N}=0
$$

it follows from (2.23) that in the low- $k$ limit $F(\boldsymbol{k}, t)$ asymptotes to $S(\boldsymbol{k})$,

$$
F(\boldsymbol{k}, t)=1+n \int(g(\boldsymbol{r})-1) \mathrm{e}^{\mathrm{i} k \cdot \boldsymbol{r}} \mathrm{d} \boldsymbol{r}+O\left(k^{2}\right)=S(\boldsymbol{k})+O\left(k^{2}\right),
$$

where $g(\boldsymbol{r})$ is the steady pair-distribution function defined by $P_{1 \mid 1}^{0}=n g(\boldsymbol{r})$ and $S(\boldsymbol{k})$ is the non-equilibrium static structure factor. In the derivation of (2.24) we have ignored the delta-function term $N \delta(\boldsymbol{k})$; this Fourier transform of the large scattering volume $\mathscr{V}$ is irrelevant to our subsequent analysis (see Rallison \& Hinch 1986).

Quantities are scaled as $\boldsymbol{r} \sim a, \boldsymbol{U} \sim \dot{\gamma} a, \boldsymbol{k} \sim a^{-1}, \boldsymbol{D} \sim D_{0}$ and $t \sim a^{2} / D_{0}$, and the Péclet number is defined as $P e=\dot{\gamma} a^{2} / D_{0}$, where $\dot{\gamma} \equiv(\dot{\boldsymbol{\Gamma}}: \dot{\boldsymbol{\Gamma}})^{1 / 2}$. We do not employ alternative symbols to denote dimensionless quantities and all quantities are dimensionless unless stated otherwise in the following. By comparing with (2.6) the short-time effective transport properties may be determined by considering their

$\dagger$ It will be shown later that velocity fluctuations cannot be added in the same pairwise manner, i.e. $\boldsymbol{U}_{i}^{\prime}=\sum_{j \neq i}^{N} \boldsymbol{U}_{i j}^{\prime}$, since it would lead to divergence of the resulting integral. 
contribution to the initial value of the logarithmic derivative $\partial_{t} \log F$,

$\partial_{t} \log F(\boldsymbol{k}, 0)=P e \boldsymbol{k} \cdot \dot{\boldsymbol{\Gamma}} \cdot \nabla_{k} \ln F-P e\left(N S_{0}\right)^{-1}\left\langle\widehat{n} \boldsymbol{k} \cdot \dot{\boldsymbol{\Gamma}} \cdot \nabla_{k} \widehat{n}^{*}\right\rangle+P e \mathrm{i} \boldsymbol{k} \cdot \boldsymbol{U}^{*}$

$$
\begin{aligned}
& -\frac{1}{S_{0}} k^{2}-\frac{1}{S_{0}} \boldsymbol{k} \boldsymbol{k}: \frac{1}{(N-2) !} \int\left(\boldsymbol{D}_{11}^{\prime}+\boldsymbol{D}_{21} \mathrm{e}^{\mathrm{i} k \cdot \boldsymbol{r}_{2}}\right) P^{0} \mathrm{~d} \boldsymbol{r}^{N} \\
& +\frac{P e}{S_{0}} \mathrm{i} \cdot \frac{1}{(N-2) !} \int \boldsymbol{U}_{2}^{\prime}\left(\mathrm{e}^{\mathrm{i} k \cdot \boldsymbol{r}_{2}}-1\right) P^{0} \mathrm{~d} \boldsymbol{r}^{N} \\
& -\frac{1}{S_{0}} \mathrm{i} \boldsymbol{k} \cdot \frac{1}{(N-2) !} \int\left[\left(\boldsymbol{D}_{22}-\boldsymbol{D}_{21}\right)\left(\mathrm{e}^{\mathrm{i} k \cdot \boldsymbol{r}_{2}}-1\right)\right] P^{0} \cdot \nabla_{2}\left[\ln P^{0}+V\right] \mathrm{d} \boldsymbol{r}^{N} \\
& -\frac{1}{S_{0}} i \boldsymbol{k} \cdot \frac{1}{(N-3) !} \int\left(\boldsymbol{D}_{23}-\boldsymbol{D}_{21}\right) \mathrm{e}^{\mathrm{i} \boldsymbol{k} \cdot \boldsymbol{r}_{2}} P^{0} \cdot \nabla_{3}\left[\ln P^{0}+V\right] \mathrm{d} \boldsymbol{r}^{N},
\end{aligned}
$$

where $P^{0} \equiv P_{N-1 \mid 1}^{0}$ to simplify notation and $S_{0} \equiv S(0)=1+n \int(g(\boldsymbol{r})-1) \mathrm{d} \boldsymbol{r}$. The shorttime or initial collective velocity, giving the $O(k)$ term in (2.25), is

$$
\boldsymbol{U}_{0}^{c} \equiv \boldsymbol{U}^{*}-\frac{1}{S_{0}} \frac{1}{(N-3) !} \int\left(\boldsymbol{D}_{23}-\boldsymbol{D}_{21}\right) P^{0} \cdot \nabla_{3}\left[\ln P^{0}+V\right] \mathrm{d} \boldsymbol{r}^{N} .
$$

If the initial distribution is the equilibrium one, then the integral in (2.26) is zero. This integral is likely to be zero for most flows, based on symmetry arguments.

Expanding the exponential $\mathrm{e}^{\mathrm{i} k \cdot \boldsymbol{r}_{2}}$ and identifying the terms quadratic in $\boldsymbol{k}$, the initial collective diffusivity is found from (2.25) at $k \rightarrow 0$ as

$$
\begin{aligned}
\boldsymbol{D}_{0}^{c} \approx & \frac{1}{S_{0}} \boldsymbol{I}+\frac{1}{S_{0}} \frac{1}{(N-2) !} \int\left[\boldsymbol{D}_{11}^{\prime}+\boldsymbol{D}_{21}\right] P^{0} \mathrm{~d} \boldsymbol{r}^{N}-\mathscr{F}_{k k} \\
& -\frac{1}{S_{0}} \frac{1}{(N-2) !} \int \boldsymbol{r}_{2}\left(\boldsymbol{D}_{22}-\boldsymbol{D}_{21}\right) P^{0} \cdot \nabla_{2}\left[\ln P^{0}+V\right] \mathrm{d} \boldsymbol{r}^{N} \\
& -\frac{1}{S_{0}} \frac{1}{(N-3) !} \int \boldsymbol{r}_{2}\left(\boldsymbol{D}_{23}-\boldsymbol{D}_{21}\right) P^{0} \cdot \nabla_{3}\left[\ln P^{0}+V\right] \mathrm{d} \boldsymbol{r}^{N},
\end{aligned}
$$

where $\mathscr{\mathscr { T }}_{k k}$ is the $k^{2}$ tensorial coefficient of the small- $k$ expansion of the velocity fluctuation integral - the term on the right-hand side of (2.25) containing $\boldsymbol{U}_{2}^{\prime}$. In deriving (2.27) we have expanded the factor $\mathrm{e}^{\mathrm{i} k \cdot r_{2}}$ for small $k$ (except for the velocity fluctuation integral) assuming that all integrals over $\boldsymbol{r}^{N}$ are convergent.

At long times, the perturbation $f_{N}$ is $O(k)$ due to the long-wave nature of the response and can be written as $f_{N}=\mathrm{i} \boldsymbol{k} \cdot \boldsymbol{b}_{N}$, where $\boldsymbol{b}_{N}\left(\boldsymbol{r}^{N}, t\right)$ is a vector function independent of $\boldsymbol{k}$ (Morris \& Brady 1996; Brady \& Morris 1997). Substituting this ansatz into (2.22) we find that the long-time collective velocity is unchanged,

$$
\boldsymbol{U}_{\infty}^{c}=\boldsymbol{U}_{0}^{c},
$$

while the long-time collective diffusivity differs from its initial value only in the terms involving third-particle effects,

$$
\begin{aligned}
\boldsymbol{D}_{\infty}^{c}=\boldsymbol{D}_{0}^{c}-\frac{1}{S_{0}} \frac{1}{(N-3) !} \int \boldsymbol{b}_{N}\left(\boldsymbol{D}_{23}-\boldsymbol{D}_{21}\right) \cdot \nabla_{3}\left[\ln P^{0}+V\right] \mathrm{d} \boldsymbol{r}^{N} \\
\quad-\frac{1}{S_{0}} \frac{1}{(N-3) !} \int\left(\boldsymbol{D}_{23}-\boldsymbol{D}_{21}\right) P^{0} \cdot \nabla_{3} \boldsymbol{b}_{N} \mathrm{~d} \boldsymbol{r}^{N} .
\end{aligned}
$$

We conclude that when hydrodynamic interactions are neglected, there is no change in the collective diffusivity with time for an arbitrary Péclet number and particle 
concentration. In the following, we therefore omit the subscripts ' 0 ' and ' $\infty$ ' of $\boldsymbol{D}^{c}$ for simplicity.

\subsection{No hydrodynamic interactions}

In this section, we develop results given in general form above for the case in which the particles have no hydrodynamic interactions. Results are developed completely (to numerical values with appropriate tensor form) for the low- and high-Pe limits. Following from the point just made at the end of $\S 2.1$, we see that in the absence of hydrodynamic interactions between particles, the long-time collective diffusivity equals its initial value, and (2.27) reduces to

$$
\boldsymbol{D}^{c}=\frac{1}{S_{0}}\left[\boldsymbol{I}-\frac{1}{(N-2) !} \int \boldsymbol{r}_{2} P^{0} \nabla_{2}\left[\ln P^{0}+V\right] \mathrm{d} \boldsymbol{r}^{N}\right] .
$$

The influence of flow is found in the $P e$-dependent structure, i.e. via both $P^{0}$ and $S_{0}$. Referring to (2.27), we see that third-particle effects enter through the final integral which vanishes when hydrodynamic interactions are neglected, and thus (2.30) is not simply a pair-level result. We can further write

$$
\frac{1}{(N-2) !} \int \boldsymbol{r}_{2} P^{0} \nabla_{2}\left[\ln P^{0}+V\right] \mathrm{d} \boldsymbol{r}^{N}=n \int \boldsymbol{r} g(\boldsymbol{r}) \nabla\left[\ln g(\boldsymbol{r})+\langle V\rangle_{2}^{0}\right] \mathrm{d} \boldsymbol{r},
$$

where

$$
\langle V\rangle_{2}^{0}=\frac{1}{(N-2) !} \int V P_{N-2 \mid 2}^{0}\left(\boldsymbol{r}_{3}, \ldots, \boldsymbol{r}_{N} \mid \boldsymbol{r}_{2}\right) \mathrm{d} \boldsymbol{r}_{3} \cdots \mathrm{d} \boldsymbol{r}_{N}
$$

is the potential of mean force averaged over the conditional probability $P_{N-2 \mid 2}^{0}$ of finding $N-2$ particles at $\boldsymbol{r}_{3}, \ldots, \boldsymbol{r}_{N}$, given that there is a particle at $\boldsymbol{r}_{2}$. Hence, the collective diffusivity becomes

$$
\boldsymbol{D}^{c}=\frac{1}{S_{0}}\left[\boldsymbol{I}-\phi \frac{3}{4 \pi} \int \boldsymbol{r} g(\boldsymbol{r}) \nabla\left[\ln g(\boldsymbol{r})+\langle V\rangle_{2}^{0}\right] \mathrm{d} \boldsymbol{r}\right]
$$

which is valid for arbitrary $P e$ and particle volume fraction $\phi=\frac{4}{3} \pi a^{3} n$. At thermodynamic equilibrium the particles are Boltzmann distributed, $g^{e q}=\exp \left(-\langle V\rangle_{2}^{0}\right)$, and the equilibrium collective diffusivity is isotropic and is (in the dimensional form)

$$
D_{e q}^{c}=\frac{D_{0}}{S^{e q}(0)}=(6 \pi \eta a)^{-1} \frac{\partial \Pi}{\partial n},
$$

as it should be for non-hydrodynamically interacting particles. To make progress in the general case, i.e. out of equilibrium, (2.31) can be simplified in the dilute limit, where for hard spheres $g^{e q}(\boldsymbol{r})=1$ at $|\boldsymbol{r}|>2$ and $\langle V\rangle_{2}^{0}=0$. After integration by parts, (2.31) becomes

$$
\boldsymbol{D}^{c}=\frac{1}{S_{0}}\left[\boldsymbol{I}+\boldsymbol{I} \phi \frac{3}{4 \pi} \int_{r>2}(g(\boldsymbol{r})-1) \mathrm{d} \boldsymbol{r}+\phi \frac{6}{\pi} \oint_{r=2} \boldsymbol{n n}(g(2)-1) \mathrm{d} \Omega\right],
$$

where $\boldsymbol{n}=-\boldsymbol{r} / 2$ is the outer unit normal vector to the surface of contact, $r=2$, and $g(2)$ denotes the contact value of the pair-distribution function (which has angular dependence on the solid angle $\Omega)$. We have used $\nabla g(\boldsymbol{r})=\nabla(g(\boldsymbol{r})-1)$ in order to neglect the integral at infinity. The first term plus first integral on the right of (2.33) can be written in terms of the static structure factor deviation from its equilibrium 
value at $k=0$ as

$$
\boldsymbol{D}^{c}=\frac{1}{S_{0}}\left[\boldsymbol{I}\left(1+\Delta S_{0}\right)+\phi \frac{6}{\pi} \oint \boldsymbol{n} \boldsymbol{n}(g(2)-1) \mathrm{d} \Omega\right],
$$

where $\Delta S_{0}=S_{0}-S_{0}^{e q}$ and $S_{0}^{e q} \sim 1-8 \phi$. Note that at equilibrium, $g(2)=1, \Delta S_{0}=0$ and thus (2.32) is recovered.

\section{High Péclet number}

For linear flows at large Péclet number, the asymptotic solution for the pairdistribution function $g(\boldsymbol{r})$ obtained in the radial balance approximation (Brady \& Morris 1997) gives contact value of

$$
g(2) \sim-\frac{2}{3} P e \gamma_{r}+O(1), \quad \text { for } \quad \gamma_{r}=\boldsymbol{n} \cdot \hat{\boldsymbol{E}} \cdot \boldsymbol{n}<0,
$$

where $P e \gg 1$ and $\hat{E}_{i j}=\left(\dot{\Gamma}_{i j}+\dot{\Gamma}_{j i}\right) / 2 \dot{\gamma}$ is the dimensionless rate-of-strain tensor. Length is scaled with the sphere radius $a$. It follows from (2.34) that for a dilute suspension

$$
\boldsymbol{D}^{c}=\frac{1}{S_{0}}\left(1+\Delta S_{0}\right) \boldsymbol{I}-\phi P e \frac{4}{\pi} \oint_{\gamma_{r}<0} \gamma_{r} \boldsymbol{n} \boldsymbol{n} \mathrm{d} \Omega+O(1) .
$$

Although the shear-induced diffusion enhancement given by the integral in (2.36) can be calculated for an arbitrary linear flow, the asymptotic evaluation of $S(0)$ in the large- $P$ e limit is a formidable task even without hydrodynamic interactions. While the radial balance approximation can be exploited to determine the leadingorder solution for $g(\boldsymbol{r})$ in the compressional quadrants, it is not applicable in the extensional quadrants $\left(\gamma_{r}>0\right)$, where low- $g$ wake-like regions extend from the surface of contact to some large distance $r \sim L(P e)$ downstream where they are smeared by a weak transverse diffusion. In the compressional quadrants $g(\boldsymbol{r}) \sim P e$ in the vicinity of the surface of contact (2.35) while the thickness of the radial boundary layer is $\sim P e^{-1}$. Therefore, the boundary layer at small $\phi$ contributes the value of $O(1)$ to the volume integral of $(g-1)$ and its positive contribution to the $\Delta S_{0}$ is estimated as $O(\phi)$ at most. On the other hand, the long low- $g$ wakes in the extensional quadrants may diminish the value of $S_{0}$ considerably due to the flow-induced 'excluded volume' effect. The microstructural deformation at large $P e$ was determined numerically by expanding $g(\boldsymbol{r})$ into spherical harmonics and integrating $(g-1)$ in the entire domain. The negative (i.e. 'excluded volume') contribution of wake-like regions to $\Delta S_{0}$ in the simple shear flow, was estimated to be of $O(\phi \sqrt{\mathrm{Pe}})$ (see the Appendix for details).

Since for the dilute suspension $S_{0}=1-8 \phi+\Delta S_{0}$, one can re-write the first term in (2.36) as

$$
\frac{1}{S_{0}}\left(1+\Delta S_{0}\right)=1+\frac{8 \phi}{1+\Delta S_{0}}
$$

the effect of the suspension microstructure deformation on the collective diffusivity is always small and the second term in (2.36), which scales linearly with $P e$, yields the leading-order contribution to $\boldsymbol{D}^{c}$.

Previous calculations by Brady \& Morris (1997) showed that the dimensional long-time self-diffusivity for the same problem is

$$
\boldsymbol{D}_{\infty}^{s}=D_{0} \boldsymbol{I}-\dot{\gamma} a^{2} \phi \frac{2}{3 \pi} \oint_{\gamma_{r}<0} \gamma_{r} \boldsymbol{n} \boldsymbol{n} \mathrm{d} \Omega .
$$


Comparing only the leading high-Péclet-number terms gives

$$
\boldsymbol{D}^{c}=6 \boldsymbol{D}_{\infty}^{s}=-\frac{a^{2}}{\eta} \frac{2}{9} \frac{\partial \boldsymbol{\Sigma}^{\prime}}{\partial \phi}
$$

where $\Sigma^{\prime}$ is the contribution to the bulk stress from contact integrals in the compressional quadrants, $\gamma_{r}<0$ :

$$
\boldsymbol{\Sigma}^{\prime}=\eta \dot{\gamma} \phi^{2} \frac{9}{\pi} \oint_{\gamma_{r}<0} \gamma_{r} \boldsymbol{n n} \mathrm{d} \Omega
$$

For example, in the simple shear flow, $\dot{\Gamma}_{i j}=\delta_{i 1} \delta_{2_{j}}$, we have in the dimensional form

$$
D_{11}^{c}=D_{22}^{c}=2 D_{33}^{c}=\frac{32}{15 \pi} \dot{\gamma} a^{2} \phi
$$

Low Péclet number

At low Péclet number the pair-distribution function can be written $g(\boldsymbol{r})=g^{e q}(r)[1+$ $p(\boldsymbol{r})]$, where $p$ is the entire $P e$-dependent perturbation to the equilibrium microstructure. For a dilute suspension, $g^{e q}=1$, and we have, in place of (2.34),

$$
\boldsymbol{D}^{c}=\frac{1}{S_{0}}\left[\left(1+\Delta S_{0}\right) \boldsymbol{I}+\phi \frac{6}{\pi} \oint \boldsymbol{n} \boldsymbol{n} p(2) \mathrm{d} \Omega\right] .
$$

Here the shear-induced deformation of the static structure factor from its equilibrium value can be written as

$$
S_{0}-S_{0}^{e q}=\Delta S_{0}=\phi \frac{3}{4 \pi} \int_{r>2} p(\boldsymbol{r}) \mathrm{d} \boldsymbol{r}+O\left(\phi^{2}\right) .
$$

In Brady \& Vicic (1995), the asymptotic expansion of $p$ to $O\left(P e^{2}\right)$ has been determined with and without hydrodynamic interactions between particles. Without hydrodynamic interactions, the contact value of $p$ is given by

$$
p(2)=-P e \frac{2}{3} \boldsymbol{n} \cdot \hat{\boldsymbol{E}} \cdot \boldsymbol{n}+P e^{2}\left(\frac{8}{27} \boldsymbol{n} \cdot \hat{\boldsymbol{E}} \cdot \hat{\boldsymbol{\Omega}} \cdot \boldsymbol{n}+\frac{8}{63} \boldsymbol{n} \cdot \hat{\boldsymbol{E}} \cdot \hat{\boldsymbol{E}} \cdot \boldsymbol{n}+\frac{128}{945} \hat{\boldsymbol{E}}: \hat{\boldsymbol{E}}\right)+O\left(P e^{5 / 2}\right),
$$

where $\hat{\Omega}_{i j}=\left(\dot{\Gamma}_{i j}-\dot{\Gamma}_{j i}\right) / 2 \dot{\gamma}$ is the dimensionless bulk vorticity tensor of the imposed linear flow and the notation $n_{i} \hat{E}_{i k} \hat{\Omega}_{k j} n_{j}$ for the first term in brackets is employed. Since the static structure factor can be written as $S_{0}=1-8 \phi+\Delta S_{0}+O\left(\phi^{2}\right)$, and in a weakly sheared suspension $\Delta S_{0}=o(\phi)$ (see the Appendix), the first term in (2.39) can be re-written as

Therefore $\boldsymbol{D}^{c}$ becomes

$$
\frac{1}{S_{0}}\left(1+\Delta S_{0}\right) \boldsymbol{I}=1+8 \phi+O\left(\phi^{2}\right)
$$

$$
\begin{aligned}
\boldsymbol{D}^{c}= & (1+8 \phi) \boldsymbol{I}-\phi \operatorname{Pe} \frac{32}{15} \hat{\boldsymbol{E}} \\
& +\phi P e^{2}\left\{\frac{128}{135} \hat{\boldsymbol{E}} \cdot \hat{\boldsymbol{\Omega}}+\frac{128}{315} \hat{\boldsymbol{E}} \cdot \hat{\boldsymbol{E}}+\frac{1024}{945}(\hat{\boldsymbol{E}}: \hat{\boldsymbol{E}}) \boldsymbol{I}\right\}+O\left(\phi P e^{5 / 2}, \phi^{2}\right) .
\end{aligned}
$$

While a uniformly valid asymptotic expansion of $p(\boldsymbol{r})$ beyond $O\left(P e^{2}\right)$ may include terms with non-integer powers of $P e$ due to non-negligible convection effects, it was found that the 'outer' solution is slaved to the 'inner' expansion, and thus the contact value $p(2)$ possesses an expansion in integer powers of $P e$ up to $O\left(P e^{5 / 2}\right)$. 


\subsection{Hydrodynamic interactions}

We now turn to the more difficult problem of evaluating the collective diffusivity with hydrodynamic interactions. We shall only consider the analytically tractable case of a weakly sheared suspension, i.e. the low-Péclet-number limit. In the work we make use of certain prior results on the structural distortion induced by weak shear flow. The added complexity of hydrodynamics in the dilute theory is largely the result of long-range hydrodynamic interactions, with expected effects on the integrals of velocity fluctuations. Nonetheless, results will be found to be qualitatively similar to the results in the absence of hydrodynamics presented in the previous section, with one important exception being a weakly divergent (as $\log k$ for $k \rightarrow 0$ ) diffusivity with tensor form given by the rate of strain, and hence having an impact only on the off-diagonal diffusivity terms in simple shear flow.

From (2.27) after neglecting the direct third-particle effects we have

$$
\begin{aligned}
\boldsymbol{D}^{c}=\frac{1}{S_{0}}\left[\boldsymbol{I}+\phi \frac{3}{4 \pi} \int\left(\boldsymbol{D}_{11}^{\prime}\right.\right. & \left.\left.+\boldsymbol{D}_{21}\right) \mathrm{~d} \boldsymbol{r}\right]+\phi \frac{3}{4 \pi} \frac{1}{S_{0}}\left[\int\left(\boldsymbol{D}_{11}^{\prime}+\boldsymbol{D}_{21}\right) p \mathrm{~d} \boldsymbol{r}\right. \\
& \left.-\int \boldsymbol{r}\left(\boldsymbol{D}_{22}-\boldsymbol{D}_{21}\right) \cdot \nabla p \mathrm{~d} \boldsymbol{r}\right]-\mathscr{F}_{k k}+O\left(\phi^{2}\right),
\end{aligned}
$$

where $p(\boldsymbol{r})$ denotes the entire $P e$-dependent uniformly valid perturbation to the equilibrium microstructure, and $\mathscr{F}_{k k}$ represents the $O\left(k^{2}\right)$ tensorial coefficient of the small- $k$ expansion of the velocity fluctuation integral:

$$
\phi P e \frac{3}{4 \pi} \frac{1}{S_{0}} \mathrm{i} \boldsymbol{k} \cdot \int_{r>2} \boldsymbol{U}_{2}^{\prime}\left(\mathrm{e}^{\mathrm{i} \boldsymbol{k} \cdot \boldsymbol{r}}-1\right) \mathrm{d} \boldsymbol{r} .
$$

The integral in the first square brackets in (2.42) is not convergent as it represents the sedimentation velocity and must be properly renormalized, as shown by Batchelor (1976). Together with the excluded volume $O(\phi)$ part of $S_{0}$, the first square brackets give the equilibrium collective diffusivity,

$$
\boldsymbol{D}_{e q}^{c}(P e=0)=\frac{1}{1-8 \phi}\left[\boldsymbol{I}+\phi \frac{3}{4 \pi} \int\left(\boldsymbol{D}_{11}^{\prime}+\boldsymbol{D}_{21}\right) \mathrm{d} \boldsymbol{r}\right]=\boldsymbol{I}(1+1.47 \phi)+O\left(\phi^{2}\right) .
$$

For a weakly sheared dilute suspension, $S_{0}=1-8 \phi+\Delta S_{0}+O\left(\phi^{2}\right)$, and the first square brackets in (2.42) can be rewritten as

$$
\frac{1}{S_{0}}\left[\boldsymbol{I}+\phi \frac{3}{4 \pi} \int\left(\boldsymbol{D}_{11}^{\prime}+\boldsymbol{D}_{21}\right) \mathrm{d} \boldsymbol{r}\right]=\boldsymbol{I}(1+1.47 \phi)-\boldsymbol{I} \Delta S_{0}+O\left(\phi^{2}\right) .
$$

The third integral on the right-hand side of (2.42) can be integrated by parts to give

$$
\begin{aligned}
\int \boldsymbol{r}\left(\boldsymbol{D}_{22}-\boldsymbol{D}_{21}\right) \cdot \nabla p \mathrm{~d} \boldsymbol{r}= & -8 \oint_{r=2} \boldsymbol{n} \cdot\left(\boldsymbol{D}_{22}-\boldsymbol{D}_{21}\right) \boldsymbol{n} p(2) \mathrm{d} \Omega \\
& +\oint_{S_{\infty}} \boldsymbol{n} \cdot\left(\boldsymbol{D}_{22}-\boldsymbol{D}_{21}\right) \boldsymbol{n} \boldsymbol{r}^{3} p(\boldsymbol{r}) \mathrm{d} \Omega \\
& -\int p \boldsymbol{r} \nabla \cdot\left(\boldsymbol{D}_{22}-\boldsymbol{D}_{21}\right) \mathrm{d} \boldsymbol{r}-\int\left(\boldsymbol{D}_{22}^{\prime}-\boldsymbol{D}_{21}\right) p \mathrm{~d} \boldsymbol{r} \\
& -\boldsymbol{I} \int p \mathrm{~d} \boldsymbol{r},
\end{aligned}
$$

where we have expanded the scaled isolated-particle diffusivity as $\boldsymbol{D}_{22}=\boldsymbol{I}+\boldsymbol{D}_{22}^{\prime}$. The first integral is zero since $\boldsymbol{D}_{22}-\boldsymbol{D}_{21}=\boldsymbol{D}_{r} / 2$ and the relative diffusivity, $\boldsymbol{D}_{r}$, vanishes 
at contact. The surface integral at infinity is also zero, as $p$ decays exponentially at large $r \gg P e^{-1 / 2}$ almost everywhere.

Finally, the integral containing $\boldsymbol{D}_{22}^{\prime}$ in (2.46) can be combined with that of $\boldsymbol{D}_{11}^{\prime}$ in (2.42) to give

$$
\begin{array}{r}
\int\left(\boldsymbol{D}_{11}^{\prime}+\boldsymbol{D}_{21}\right) p \mathrm{~d} \boldsymbol{r}-\int \boldsymbol{r}\left(\boldsymbol{D}_{22}-\boldsymbol{D}_{21}\right) \cdot \nabla p \mathrm{~d} \boldsymbol{r}=2 \int \boldsymbol{D}_{11}^{\prime} p \mathrm{~d} \boldsymbol{r} \\
+\frac{1}{2} \int p \boldsymbol{r} \nabla \cdot \boldsymbol{D}_{r} \mathrm{~d} \boldsymbol{r} \\
+\boldsymbol{I} \int p \mathrm{~d} \boldsymbol{r} .
\end{array}
$$

All integrals in (2.47) are absolutely convergent. Moreover, the regular asymptotic expansion of $p$ up to $O\left(P e^{2}\right)$ (Brady \& Vicic 1995) can be used in the first two integrals since $\boldsymbol{D}_{11}^{\prime} \sim r^{-4}$ and $\nabla \cdot \boldsymbol{D}_{r} \sim r^{-5}$, while $p_{1} \sim r^{-3}$ and $p_{2} \sim r^{-1}$. The last term in (2.47), if multiplied by $(3 / 4 \pi) \phi / S_{0}$, and using (2.40) gives $I \Delta S_{0}+o(\phi)$. This term requires the uniformly valid expansion of $p$ (see Appendix A), but fortunately, it cancels out exactly with the last term in (2.45) and $\boldsymbol{D}_{0}^{c}$ up to $O\left(P e^{2}\right)$ can be calculated through the regular asymptotic expansion of $p$. Hence, the resulting gradient diffusivity (2.42) will have the form

$$
\boldsymbol{D}^{c}=\boldsymbol{I}(1+1.47 \phi)+\phi \frac{3}{4 \pi}\left[2 \int \boldsymbol{D}_{11}^{\prime} p \mathrm{~d} \boldsymbol{r}+\frac{1}{2} \int p \boldsymbol{r} \nabla \cdot \boldsymbol{D}_{r} \mathrm{~d} \boldsymbol{r}\right]-\mathscr{F}_{k k}+O\left(\phi^{2}\right) .
$$

Now we turn to the velocity fluctuation integral (2.43) which is divergent if hydrodynamic interactions are considered in a pairwise additive manner. Expanding the phase factor $\mathrm{e}^{\mathrm{i} k \cdot \boldsymbol{r}}$ for small $k$ leads to $\mathscr{F}_{k k}$ in terms of the integral of $\boldsymbol{U}_{2}^{\prime} \boldsymbol{r}$ over the whole space, and for neutrally buoyant spheres in linear flows $\boldsymbol{U}_{2}^{\prime}=$ $\left(\boldsymbol{U}_{2}-\boldsymbol{U}_{1}\right)-\dot{\boldsymbol{\Gamma}} \cdot \boldsymbol{r} \sim \boldsymbol{r}^{-2}$. Therefore, a small-k expansion under the integral sign in (2.43) leads to a divergent integral. The divergence of ensemble averages due to long-range hydrodynamic interactions in unbounded suspensions is well-studied (Batchelor 1972; Hinch 1977). Thus, the velocity fluctuation integral needs to be re-normalized in the standard fashion for non-convergent hydrodynamic interactions. Note that this term would be non-convergent even if we had not expanded $\mathrm{e}^{\mathrm{i} k \cdot \boldsymbol{r}}$ (meaning it is not the weighting with $\boldsymbol{r}$ that causes difficulty), and we cannot simply add velocity fluctuations in a pairwise manner. Following Brady et al. (1988), we write the velocity of particle 2 due to the motion of particle 1 averaged over all positions of particle 2 to obtain

$$
\mathrm{i} \boldsymbol{k} \cdot \int_{r>2}\left(\boldsymbol{U}_{2}^{\prime}-\boldsymbol{U}_{2}^{\prime, \infty}\right)\left(\mathrm{e}^{\mathrm{i} \boldsymbol{k} \cdot \boldsymbol{r}}-1\right) \mathrm{d} \boldsymbol{r}+\boldsymbol{k} \boldsymbol{k}: \int_{r<2} \boldsymbol{r} \boldsymbol{U}_{2}^{\prime, \infty} \mathrm{d} \boldsymbol{r}
$$

where we have expanded the exponential in the last integral. This integral arises from the 'backflow' being defined over the whole space, not just that outside the particle at $r=2$. Here, $\boldsymbol{U}_{2}^{\prime, \infty}$ is the far-field part of the velocity disturbance that decays as $r^{-2}$. The difference $\boldsymbol{U}_{2}^{\prime}-\boldsymbol{U}_{2}^{\prime, \infty}$ decays as $r^{-4}$ and the integral is absolutely convergent. In this case we cannot simply expand the exponential in the first term as the $\boldsymbol{r}$ weighting results in a conditionally convergent integral. We can, however, extract terms that decay faster than $r^{-4}$ by defining

$$
\boldsymbol{U}_{2}^{\prime}-\boldsymbol{U}_{2}^{\prime, \infty}=\boldsymbol{U}_{2}^{\prime, 0}-\boldsymbol{U}_{2}^{\prime, \infty}+\boldsymbol{U}_{2}^{\prime \prime},
$$


where $\boldsymbol{U}_{2}^{\prime, 0}$ contains terms up to $r^{-4}$ and $\boldsymbol{U}_{2}^{\prime \prime}$ is the remainder. Thus, the velocity fluctuation integral becomes finally

$$
\mathrm{i} \boldsymbol{k} \cdot \int_{r>2}\left(\boldsymbol{U}_{2}^{\prime, 0}-\boldsymbol{U}_{2}^{\prime, \infty}\right)\left(\mathrm{e}^{\mathrm{i} \boldsymbol{k} \cdot \boldsymbol{r}}-1\right) \mathrm{d} \boldsymbol{r}+\boldsymbol{k} \boldsymbol{k}: \int_{r<2} \boldsymbol{r} \boldsymbol{U}_{2}^{\prime, \infty} \mathrm{d} \boldsymbol{r}-\boldsymbol{k} \boldsymbol{k}: \int_{r>2} \boldsymbol{r} \boldsymbol{U}_{2}^{\prime \prime} \mathrm{d} \boldsymbol{r},
$$

where all integrals are absolutely convergent. Other than the integral involving the $r^{-4}$ dependence of $\boldsymbol{U}_{2}^{\prime}$, the integrations to evaluate $D^{c}$ are straightforward. This integral may be written

$$
\begin{aligned}
\mathrm{i} \boldsymbol{k} \cdot \int_{r>2} & \left(\boldsymbol{U}_{2}^{\prime, 0}-\boldsymbol{U}_{2}^{\prime, \infty}\right)\left(\mathrm{e}^{\mathrm{i} \boldsymbol{k} \cdot \boldsymbol{r}}-1\right) \mathrm{d} \boldsymbol{r} \\
& =\mathrm{i} \boldsymbol{k} \cdot \int_{r>2} \boldsymbol{r} \cdot \boldsymbol{E} \cdot\left[\left(A^{(-5)}(r)-B^{(-5)}(r)\right) \boldsymbol{n} \boldsymbol{n}+B^{(-5)}(r) \boldsymbol{l}\right] \mathrm{e}^{\mathrm{i} \boldsymbol{k} \cdot \boldsymbol{r}} \mathrm{d} \boldsymbol{r},
\end{aligned}
$$

noting that, due to symmetry, the discarded term (involving the -1 ) vanishes. Here $A^{(-5)}$ and $B^{(-5)}$ are the $r^{-5}$ far-field dependences of the hydrodynamic functions $A(r)$ and $B(r)$ specifying the radial dependence of the velocity disturbance in a pair interaction (Batchelor \& Green 1972)

$$
\left(\boldsymbol{U}_{2}-\boldsymbol{U}_{1}\right)-\dot{\boldsymbol{\Gamma}} \cdot \boldsymbol{r}=-\boldsymbol{r} \cdot \boldsymbol{E} \cdot[A(r) \boldsymbol{n} \boldsymbol{n}+B(r)(\boldsymbol{I}-\boldsymbol{n} \boldsymbol{n})],
$$

and for identical spheres of unit radius, these are

$$
A^{(-5)}=-8 r^{-5}, \quad \text { and } \quad B^{(-5)}=\frac{16}{3} r^{-5} .
$$

Substituting these values into (2.51) we arrive at

$$
\mathrm{i} k_{j} \hat{E}_{l m}\left[\frac{40}{3} \int_{r>2} \frac{n_{j} n_{l} n_{m}}{r^{4}} \mathrm{e}^{\mathrm{i} k \cdot r} \mathrm{~d} \boldsymbol{r}-\frac{16}{3} \int_{r>2} \frac{\delta_{m j} n_{l}}{r^{4}} \mathrm{e}^{\mathrm{i} k \cdot r} \mathrm{~d} \boldsymbol{r}\right] .
$$

Recognizing that the integrals over the angular dependence are proportional to $\boldsymbol{k} \boldsymbol{k} \boldsymbol{k}$ and $\boldsymbol{k} \boldsymbol{l}$, the entire result must be proportional to $\boldsymbol{k} \cdot \hat{\boldsymbol{E}} \cdot \boldsymbol{k}$. To perform the integrations we write the integrals as

$$
\begin{aligned}
\mathrm{i} k_{j} \hat{E}_{l m} \int_{r>2} \frac{\delta_{m j} n_{l}}{r^{4}} \mathrm{e}^{\mathrm{i} k \cdot r} \mathrm{~d} \boldsymbol{r} & =k_{l} \hat{E}_{l j} k_{j} \frac{\mathscr{K}^{\prime}(k)}{k}, \\
\mathrm{i} k_{j} \hat{E}_{l m} \int_{r>2} \frac{n_{j} n_{l} n_{m}}{r^{4}} \mathrm{e}^{\mathrm{i} k \cdot r} \mathrm{~d} \boldsymbol{r} & =-k_{j} \hat{E}_{l m} \frac{\partial^{3}}{\partial k_{j} \partial k_{l} \partial k_{m}} \mathscr{M}(k) \\
& =-k_{l} \hat{E}_{l m} k_{m}\left(\frac{\mathscr{M}^{\prime}}{k^{3}}-\frac{\mathscr{M}^{\prime \prime}}{k^{2}}\right),
\end{aligned}
$$

where

$$
\mathscr{K}(k)=\int_{r>2} \frac{\mathrm{e}^{\mathrm{i} k \cdot \boldsymbol{r}}}{r^{5}} \mathrm{~d} \boldsymbol{r} \quad \text { and } \quad \mathscr{M}(k)=\int_{r>2} \frac{\mathrm{e}^{\mathrm{i} k \cdot \boldsymbol{r}}}{r^{7}} \mathrm{~d} \boldsymbol{r} .
$$

The evaluation of expressions involving both these functions in (2.53)-(2.54) is straightforward and, for instance, for $\mathscr{K}$ yields

$$
\begin{aligned}
\frac{\mathscr{K}^{\prime}(k)}{k}=\frac{1}{k} \int_{r>2} \frac{\mathrm{i} \mu \mathrm{e}^{\mathrm{i} k r \mu}}{r^{4}} \mathrm{~d} \boldsymbol{r} & =\frac{4 \pi}{k^{3}} \int_{r=2}^{\infty} \frac{k r \cos k r-\sin k r}{r^{4}} \mathrm{~d} r \\
& =\frac{4 \pi}{3 \alpha^{3}}\left[\alpha \cos \alpha+\alpha^{3} \operatorname{Ci}(\alpha)-\left(1+\alpha^{2}\right) \sin \alpha\right]
\end{aligned}
$$


where $\mu$ is the cosine of the angle between $\boldsymbol{k}$ and $\boldsymbol{n}, \alpha=2 k$ and $\mathrm{Ci}$ denotes the cosine integral function. Taking the limit $k \rightarrow 0$ we find

$$
\frac{\mathscr{K}^{\prime}(k)}{k}=\frac{4 \pi}{3}\left(\ln k+\ln 2+\gamma_{E}-\frac{4}{3}\right)+O\left(k^{2}\right),
$$

where $\gamma_{E} \simeq 0.5772$ is the Euler constant. Analogously,

$$
\frac{\mathscr{M}^{\prime}}{k^{3}}-\frac{\mathscr{M}^{\prime \prime}}{k^{2}}=\frac{4 \pi}{15}\left(\ln k+\ln 2+\gamma_{E}-\frac{23}{15}\right)+O\left(k^{2}\right) .
$$

Inserting these expressions into (2.53)-(2.54) and gathering according to (2.52) we finally obtain that for small $k$

$$
\mathrm{i} \boldsymbol{k} \cdot \phi \int_{r>2}\left(\boldsymbol{U}_{2}^{\prime, 0}-\boldsymbol{U}_{2}^{\prime, \infty}\right)\left(\mathrm{e}^{\mathrm{i} k \cdot \boldsymbol{r}}-1\right) \mathrm{d} \boldsymbol{r}=-\frac{32 \pi}{3} \phi\left(\ln k+\ln 2+\gamma_{E}-\frac{7}{5}\right) \boldsymbol{k} \cdot \hat{\boldsymbol{E}} \cdot \boldsymbol{k}+O\left(k^{4}\right) .
$$

Noting that

$$
\boldsymbol{U}_{2}^{\prime, \infty}=-5 r^{-2} \boldsymbol{n} \cdot \hat{\boldsymbol{E}} \cdot \boldsymbol{n} \boldsymbol{n}=-5 r^{-2} \gamma_{r} \boldsymbol{n},
$$

the second integral in (2.50) over the excluded volume $r<2$ yields

$$
\int_{r<2} \boldsymbol{r} \boldsymbol{U}_{2}^{\prime, \infty} \mathrm{d} \boldsymbol{r}=-\frac{16 \pi}{3} \hat{\boldsymbol{E}} \text {. }
$$

The last integral in (2.50), evaluated numerically, yields

$$
\begin{aligned}
\int_{r>2} \boldsymbol{r} \boldsymbol{U}_{2}^{\prime \prime} \mathrm{d} \boldsymbol{r} & =-\hat{\boldsymbol{E}}: \oint \boldsymbol{n n n n n} \mathrm{d} \Omega \int_{2}^{\infty}\left(A^{\prime \prime}-B^{\prime \prime}\right) r^{4} \mathrm{~d} r-\hat{\boldsymbol{E}} \cdot \oint \boldsymbol{n} \boldsymbol{n} \mathrm{d} \Omega \int_{2}^{\infty} B^{\prime \prime} r^{4} \mathrm{~d} r \\
& =-\frac{8 \pi}{15} \hat{\boldsymbol{E}}(12.5)-\frac{4 \pi}{3} \hat{\boldsymbol{E}}(0.23)=-\frac{4 \pi}{3} \hat{\boldsymbol{E}}(5.23),
\end{aligned}
$$

where $A^{\prime \prime}(r)$ and $B^{\prime \prime}(r)$ are the $o\left(r^{-5}\right)$ truncated hydrodynamic functions according to (2.49). Thus, collecting all the contributions in (2.50) we finally obtain the contribution of the velocity fluctuation integral to the collective diffusivity:

$$
\begin{aligned}
\phi P e \frac{3}{4 \pi} \frac{1}{S_{0}} \mathrm{i} \boldsymbol{k} \cdot \int \boldsymbol{U}_{2}^{\prime}\left(\mathrm{e}^{\mathrm{i} \boldsymbol{k} \cdot \boldsymbol{r}}-1\right) \mathrm{d} \boldsymbol{r}= & -8 \phi P e\left(\ln k+\ln 2+\gamma_{E}-1.55\right) \boldsymbol{k} \cdot \hat{\boldsymbol{E}} \cdot \boldsymbol{k} \\
& +O\left(k^{2}, \phi^{2}\right) .
\end{aligned}
$$

Note that the abnormal scaling $\sim \ln k$ is affecting the components of $\boldsymbol{D}^{c}$ which have the geometry of the imposed flow $\hat{\boldsymbol{E}}$. Therefore, in the case of a simple shear flow it only influences the off-diagonal collective-diffusion coefficient $D_{12}^{c}\left(D_{13}^{c}=D_{23}^{c}=0\right.$ by symmetry), while the diagonal components of $D_{i i}^{c}$ are truly diffusive, as we shall see below.

To complete the asymptotic evaluation of $\boldsymbol{D}^{c}$ at $O(\mathrm{Pe})$ we also must evaluate integrals on the right-hand side of (2.47):

$$
\int_{r>2} p_{1} \boldsymbol{D}_{11}^{\prime} d \boldsymbol{r}=-\hat{\boldsymbol{E}}: \oint \boldsymbol{n n n n} \mathrm{d} \Omega \int_{2}^{\infty} q(r)\left(x_{11}^{a}(r)-y_{11}^{a}(r)\right) r^{2} \mathrm{~d} r=\frac{8 \pi}{15} \hat{\boldsymbol{E}}(0.44)
$$

and

$$
\int_{r>2} p_{1} \boldsymbol{r} \nabla \cdot \boldsymbol{D}_{r} d \boldsymbol{r}=-\hat{\boldsymbol{E}}: \oint \boldsymbol{n n n n \boldsymbol { n }} \mathrm{d} \int_{2}^{\infty} q(r) Z(r) r^{3} \mathrm{~d} r=\frac{8 \pi}{15} \hat{\boldsymbol{E}}(-2.57),
$$


where $q(r)$ is given in (A 37). In (2.61) we exploited the fact that

$$
\nabla \cdot \boldsymbol{D}_{r} \equiv \boldsymbol{n Z}(r)=\boldsymbol{n}\left\{G^{\prime}(r)+2 \frac{G(r)-H(r)}{r}\right\} .
$$

Here $x_{11}^{a}, y_{11}^{a}, Z(r), G(r)$ and $H(r)$ are known mobility functions (Jeffrey \& Onishi 1984; Kim \& Karrila 1991).

Gathering all contributions, the collective diffusivity is given to $O(\mathrm{Pe})$ by

$$
\boldsymbol{D}^{c}=(1+1.47 \phi) \boldsymbol{I}+8(\ln k+\mathscr{C}) \phi P e \hat{\boldsymbol{E}}+O\left(\phi^{2}, P e^{2}\right),
$$

where $\mathscr{C}=\ln 2+\gamma_{E}-1.64 \simeq-0.37$. Note that the coefficient of the term linear in $\hat{\boldsymbol{E}}$ is negative for small $k$, in agreement with the sign found without hydrodynamic interaction.

To complete the evaluation of $\boldsymbol{D}^{c}$ to $O\left(P e^{2}\right)$, it follows from (2.48) that we also must determine

$$
\phi P e^{2} \frac{3}{4 \pi}\left[2 \int \boldsymbol{D}_{11}^{\prime} p_{2}(\boldsymbol{r}) \mathrm{d} \boldsymbol{r}+\frac{1}{2} \int \boldsymbol{r} \nabla \cdot \boldsymbol{D}_{r} p_{2}(\boldsymbol{r}) \mathrm{d} \boldsymbol{r}+\int \boldsymbol{r} \boldsymbol{U}_{2}^{\prime} p_{1}(\boldsymbol{r}) \mathrm{d} \boldsymbol{r}\right],
$$

where the last integral comes from small- $k$ expansion of the velocity fluctuation integral (2.43). The first two integrals are convergent as discussed just after (2.47), while the last integral is convergent since $p_{1}$ decays as $r^{-3}$ for large $r$ and $\boldsymbol{U}_{2}^{\prime} \sim r^{-2}$. It is expected from the form of $p_{1}, p_{2}$ and $\boldsymbol{U}_{2}^{\prime}$ that the $O\left(P e^{2}\right)$ contribution is quadratic in the imposed flow and is a linear combination of $\hat{\boldsymbol{E}} \cdot \hat{\boldsymbol{\Omega}}, \hat{\boldsymbol{E}} \cdot \hat{\boldsymbol{E}}$ and $(\hat{\boldsymbol{E}}: \hat{\boldsymbol{E}}) \boldsymbol{I}$ as was the case without hydrodynamics (2.41). The constant coefficients are to be determined by numerical integration. The velocity fluctuation integral in component form is

$$
\begin{aligned}
\int r_{i} U_{2_{j}}^{\prime} p_{1}(\boldsymbol{r}) \mathrm{d} \boldsymbol{r}= & \hat{E}_{k l} \hat{E}_{r s} \oint n_{i} n_{j} n_{k} n_{l} n_{r} n_{s} \mathrm{~d} \Omega \int(A(r)-B(r)) q(r) r^{4} \mathrm{~d} r \\
& +\hat{E}_{j k} \hat{E}_{r s} \oint n_{i} n_{k} n_{r} n_{s} \mathrm{~d} \Omega \int B(r) q(r) r^{4} \mathrm{~d} r \\
= & 4 \pi\left[\frac{2}{105} \hat{E}_{k s} \hat{E}_{k s} \delta_{i j} \mathscr{I}_{1}+\left(\frac{8}{105} \mathscr{I}_{1}+\frac{2}{15} \mathscr{I}_{2}\right) \hat{E}_{i k} \hat{E}_{k j}\right],
\end{aligned}
$$

where the numerical evaluation of the radial integrals in the two terms on the righthand side yields $\mathscr{I}_{1}=\int_{2}^{\infty}(A(r)-B(r)) q(r) r^{4} \mathrm{~d} r=20.90$ and $\mathscr{I}_{2}=\int_{2}^{\infty} B(r) q(r) r^{4} \mathrm{~d} r=$ 2.36 , respectively, and the surface integrals are evaluated using the identity

$$
\frac{1}{4 \pi} \oint \underbrace{n_{\alpha_{1} n_{\alpha_{2}} \ldots n_{\alpha_{m}}}}_{\text {m even }} \mathrm{d} \Omega=\frac{1}{(m+1) ! !} \sum_{p} \delta_{\alpha_{1} \alpha_{2}} \delta_{\alpha_{3} \alpha_{4}} \ldots \delta_{\alpha_{m-1} \alpha_{m}},
$$

where $p$ is the number of all possible index permutations. The first two integrals in (2.63) can be written in the form

$$
\int \boldsymbol{n} \boldsymbol{n} p_{2}(\boldsymbol{r}) \mathscr{T}^{(1,2)}(r) \mathrm{d} \boldsymbol{r}
$$

where $\mathscr{T}^{(1)}=x_{11}^{a}(r)-y_{11}^{a}(r)$ and $\mathscr{T}^{(2)}=r Z(r)$ for the first and second integral, respectively. Substituting the known expansion for $p_{2}(\boldsymbol{r})$ in terms of $h_{2}(r)-h_{5}(r)$ (Brady \& Vicic 1995, Appendix) and using the above identity for integrals over the 
unit sphere, we arrive at the following result for (2.65):

$$
\begin{aligned}
& -4 \pi\left[\left(\frac{8}{105} \mathscr{J}_{2}^{(1,2)}+\frac{2}{15} \mathscr{J}_{4}^{(1,2)}\right) \hat{E}_{i k} \hat{E}_{k j}+\frac{2}{15} \mathscr{J}_{3}^{(1,2)} \hat{E}_{i k} \hat{\Omega}_{k j}\right. \\
& \left.+\left(\frac{2}{105} \mathscr{J}_{2}^{(1,2)}+\frac{1}{15} \mathscr{J}_{4}^{(1,2)}+\frac{1}{3} \mathscr{J}_{5}^{(1,2)}\right) \hat{E}_{l m} \hat{E}_{m l} \delta_{i j}\right]
\end{aligned}
$$

where

$$
\mathscr{J}_{\alpha}^{(1,2)}=\int_{r=2}^{\infty} h_{\alpha}(r) \mathscr{T}^{(1,2)}(r) r^{2} \mathrm{~d} r .
$$

Evaluating $\mathscr{J}_{\alpha}^{(1,2)}$ numerically and substituting these results together with (2.64) into (2.63) we finally obtain

$$
\begin{aligned}
\boldsymbol{D}^{c}=(1+1.47 \phi) \boldsymbol{I} & +8(\ln k+\mathscr{C}) \phi P e \hat{\boldsymbol{E}} \\
& +\phi P e^{2}\{0.063 \hat{\boldsymbol{E}} \cdot \hat{\boldsymbol{\Omega}}+5.80 \hat{\boldsymbol{E}} \cdot \hat{\boldsymbol{E}}+1.22(\hat{\boldsymbol{E}}: \hat{\boldsymbol{E}}) \boldsymbol{I}\}+O\left(\phi^{2}\right) .
\end{aligned}
$$

Note the similarity between this result and the approximate solution without hydrodynamics $(2.41)$ except for the weakly singular term linear in $\hat{\boldsymbol{E}}$. In the simple shear flow, $\dot{\Gamma}_{i j}=\delta_{i 1} \delta_{2 j}$, where the measurements and simulations are most feasible, the shear-induced enhancement of the collective diffusivity is expected to be small, $O\left(\phi P e^{2}\right)$, with the transverse components in the dimensional form given by

$$
\left(D_{22}^{c}-D_{e q}^{c}\right) / D_{0}=2.08 \phi P e^{2}, \quad\left(D_{33}^{c}-D_{e q}^{c}\right) / D_{0}=0.61 \phi P e^{2},
$$

where $D_{e q}^{c}=D_{0}(1+1.47 \phi)$.

\section{Brownian Dynamics simulations}

\subsection{Simulation method}

The long-time collective-diffusion tensor was determined using Brownian Dynamics (BD) simulations of a hard-sphere unbounded suspension in the simple shear flow, $\boldsymbol{U}^{\infty}=\dot{\gamma} y \boldsymbol{e}_{x}$. These simulations do not include hydrodynamic interactions. The simulation box is cubic with periodic boundary conditions in the three principal directions. We use an algorithm similar to one used in Foss \& Brady (1999) and based on the potential-free method of Heyes \& Melrose (1993) and Schaertl \& Sillescu (1994). The displacements of the particles are governed by the following evolution equation:

$$
\Delta \boldsymbol{x}=\Delta \boldsymbol{x}^{a}+\Delta \boldsymbol{x}^{H S}+\boldsymbol{X}(\Delta t)
$$

where $\Delta \boldsymbol{x}^{a}=\boldsymbol{U}^{\infty} \Delta t$ is the affine displacement over a time step due to imposed flow and is equal to $\dot{\gamma} y \boldsymbol{e}_{x} \Delta t$ for a simple shear flow; $\Delta \boldsymbol{x}^{H S}$ is the displacement due to a hard-sphere potential; $\boldsymbol{X}$ is the random Brownian step with zero mean and variance equal to twice the Stokes-Einstein diffusivity of a single particle, $D_{0}$,

$$
\bar{X}=0, \quad \text { and } \overline{\boldsymbol{X}(\Delta t) \boldsymbol{X}(\Delta t)}=2 D_{0} I \Delta t .
$$

After the affine and the random Gaussian contribution are added, the algorithm searches for particles pairs that have overlapped and moves each particle along their line of centres to contact in response to the 'hard-sphere-like' potential. The procedure is repeated until no overlapping sphere pairs are present and the resulting displacement vector $\Delta \boldsymbol{x}^{H S}$ is added to the first two. This is the major difference between the present algorithm and the scheme used by Foss \& Brady (1999), where 
this procedure is only called once on each time step and thus a small amount of inherent 'softness' due to three-body effects is allowed.

It has been demonstrated in Leshansky \& Brady (2005) that the collective diffusivity $\boldsymbol{D}^{c}$ of particles suspended in an unbounded linear flow can be identified through the evolution of the dynamic structure factor $F$ in (2.6). Specifically, we apply the following ansatz:

$$
\hat{\boldsymbol{k}} \cdot \mathscr{D}(\boldsymbol{k}) \cdot \hat{\boldsymbol{k}}=-\mathrm{i} \boldsymbol{k} \cdot\left(N F k^{2}\right)^{-1}\left\langle\sum_{\alpha, \beta}^{N} \boldsymbol{U}_{\alpha}^{\prime} \mathrm{e}^{\mathrm{i} \boldsymbol{k} \cdot\left(x_{\alpha}(t)-x_{\beta}(0)\right)}\right\rangle,
$$

where $\hat{\boldsymbol{k}}=\boldsymbol{k} /|\boldsymbol{k}|$. Here $\boldsymbol{U}_{\alpha}^{\prime}$ is the configuration-dependent velocity fluctuation of particle $\alpha$, i.e. the velocity of particle $\alpha$ relative to the bulk uniform and shearing motions measured at some reference point, $\boldsymbol{x}_{0}$,

$$
\boldsymbol{U}_{\alpha}=\dot{\boldsymbol{\Gamma}} \cdot\left(\boldsymbol{x}_{\alpha}-\boldsymbol{x}_{0}\right)+\boldsymbol{U}^{\infty}\left(\boldsymbol{x}_{0}\right)+\boldsymbol{U}_{\alpha}^{\prime}=\dot{\boldsymbol{\Gamma}} \cdot \boldsymbol{x}_{\alpha}+\boldsymbol{U}^{*}+\boldsymbol{U}_{\alpha}^{\prime},
$$

with $\boldsymbol{U}^{*}=\boldsymbol{U}^{\infty}-\dot{\boldsymbol{\Gamma}} \cdot \boldsymbol{x}_{0}$. The formula (3.2) describes diffusivity in an arbitrary direction; it was derived from purely kinematic considerations and therefore holds regardless of the microscopic dynamics of the system. On the other hand if one is only interested in diffusivity in the direction transverse to the direction of the undisturbed flow, i.e. for $\hat{\boldsymbol{k}} \cdot \dot{\boldsymbol{\Gamma}} \cdot \hat{\boldsymbol{k}}=\hat{\boldsymbol{k}} \cdot \boldsymbol{U}^{*}=0$ the convective terms on the left-hand side of (2.6) drop out, yielding the familiar quasi-equilibrium solution for $F$ describing exponential decay of $F(\boldsymbol{k}, t)$,

$$
-\frac{\dot{F}}{k^{2} F}=\hat{\boldsymbol{k}} \cdot \mathscr{D}_{\perp}(\boldsymbol{k}) \cdot \hat{\boldsymbol{k}}
$$

Equation (3.3) is well known for equilibrium colloidal dispersions, where in the absence of an imposed flow $\hat{\boldsymbol{k}} \cdot \mathscr{D} \cdot \hat{\boldsymbol{k}}=\mathscr{D}(k)$, with $\mathscr{D}(k)$ being a scalar due to spatial isotropy. In the simple shear flow $\mathscr{D}$ is a second-rank tensor, and the two distinct transverse collective-diffusion coefficients, $D_{y y}^{c}$ (velocity-gradient direction) and $D_{z z}^{c}$ (vorticity direction), respectively, can be extracted by probing the dynamics of $F(\boldsymbol{k}, t)$ at small $a k$.

\subsection{Results and discussion}

\section{Collective diffusion at equilibrium}

First we test the proposed approach by calculating the equilibrium long-time gradient-diffusion coefficient, $D_{e q}^{c}(P e=0)$ at different particle volume fractions $\phi=$ $0.10-0.40$. The computational efficiency of the $\mathrm{BD}$ algorithm allows for systems with a large number of particles, while in most cases we found that systems with $N=256$ particles provide accurate results for moderate concentrations up to $\phi=0.30$.

Following Sierou \& Brady (2004) and Leshansky \& Brady (2005), the values of $F$ are averaged over all possible (overlapping) time intervals available for each simulation run to reduce the statistical noise. To further improve the statistics, we average the data over 32 long runs of up to 50 time units, scaled with the diffusion time $\left(a^{2} / D_{0}\right)$, starting from independent hard-sphere configurations. Figure 1 shows the time evolution of the dynamic structure factor plotted in the form $-k^{-2} \ln F$ as a function of scaled time. Here, $F$ is calculated using (2.9) at the cutoff wavenumber $k_{c}=2 \pi / H$, where $H$ is the dimension of the cubic simulation cell, and averaged over all directions due to isotropy of the equilibrium suspension. It is evident from figure 1 that all the curves are straight lines and, therefore, in the absence of flow the linear diffusive regime is established immediately (on the time scale $\sim a^{2} / D_{0}$ ) and $F$ 


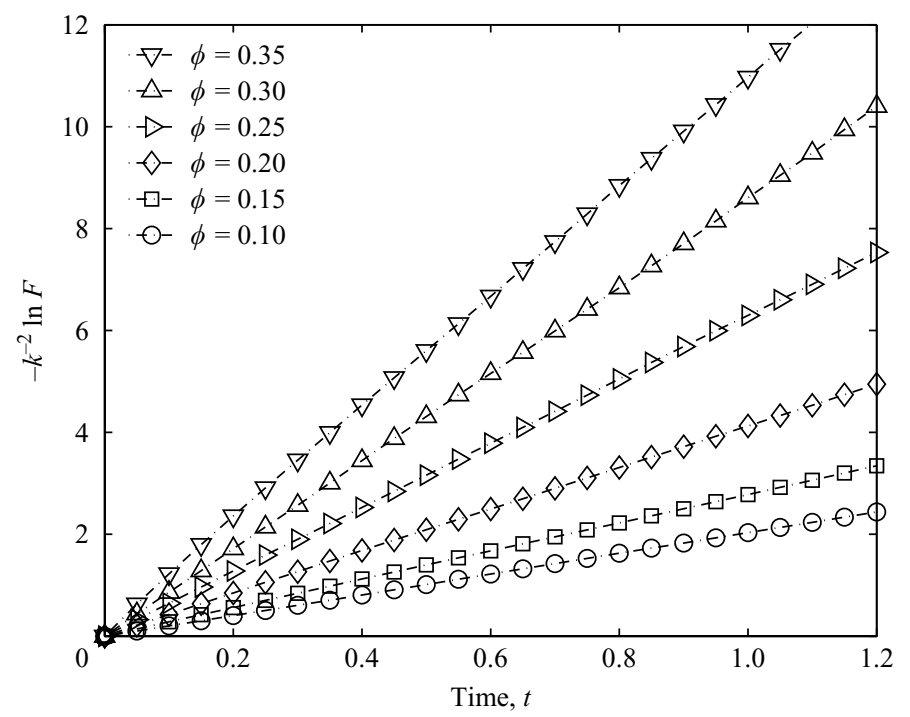

FIGURE 1. Evolution of the dynamic structure factor, $-k^{-2} \ln F$, at $P e=0$ probed at $k=2 \pi /$ $H$, vs. time scaled by $\left(a^{2} / D_{o}\right)$ for a system of $N=256$ particles and different concentrations.

decays exponentially according to (3.3). The collective diffusivity, determined as the slopes of the curves, is an increasing function of $\phi$. This observation is in contrast to the long-time self-diffusion, where increasing frequency of particle collisions hinders self-displacement of the particles.

In the absence of hydrodynamic interactions, the mobility of each particle is unaffected by the presence of other particles, $\mathscr{H}=1$, and therefore, from (1.1), the collective diffusion is driven solely by the osmotic compressibility, with the diffusivity being equal to the inverse of the equilibrium static structure factor at zero $k$,

$$
D^{c} / D_{0}=S^{e q}(0)^{-1} \text {. }
$$

The values of $S_{0}^{e q}$ can be estimated for the hard-sphere dispersion up to $\phi \approx 0.5$ from the Carnahan-Starling approximation (Russel, Saville \& Schowalter 1989),

$$
S^{e q}(0)=\frac{(1-\phi)^{4}}{1+4 \phi+4 \phi^{2}-4 \phi^{3}+\phi^{4}} .
$$

The comparison between the results of our numerical simulations from figure 1 and the analytical prediction (3.4) is presented in figure 2. There is excellent agreement between the two up to a concentration of $\sim 0.3$, while for higher volume fractions the values of $D^{c}$ from the BD simulations ( $\square$ symbols) are underestimated. Finitesystem effects are responsible for this discrepancy. Indeed, when we repeat the BD simulations for a system of $N=1024$ particles (o symbols in figure 2), there is much closer agreement for the volume fractions up to 0.40 . It is interesting to note that the static microstructure of the suspension sampled at all $k$ values appears to be wellcaptured with $N=256$ particles (see the inset in figure 2), while the time correlation remains measurably affected by the periodicity of the boundaries.

\section{Sheared suspension at arbitrary Pe}

When the suspension is sheared, the collective diffusivity in directions transverse to the direction of flow is determined in the same way as for the equilibrium case 


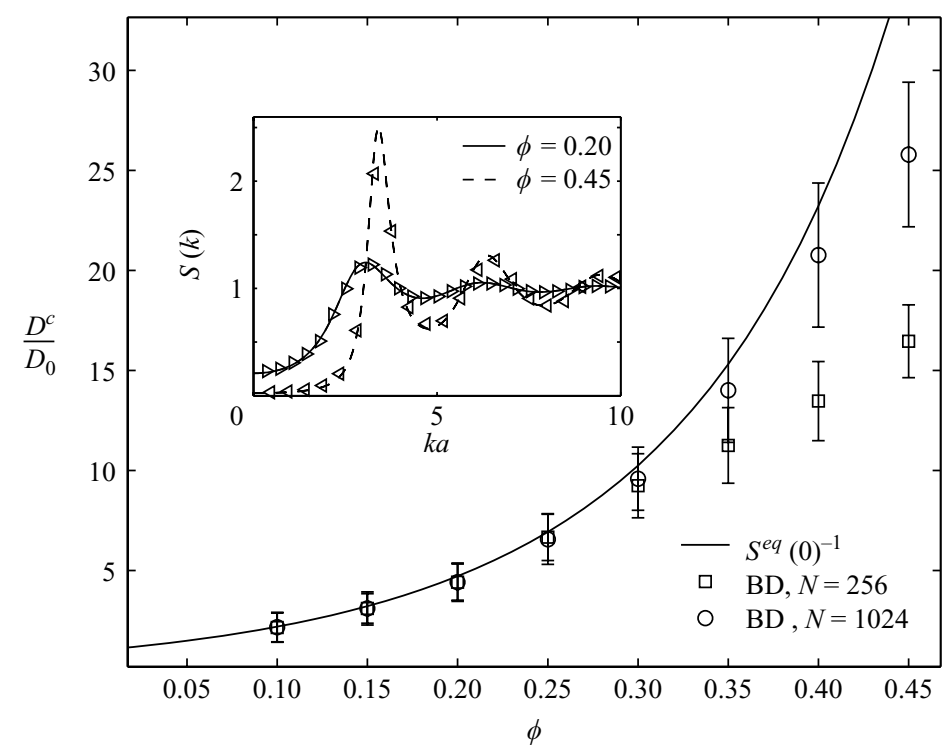

FIGURE 2. Equilibrium $(P e=0)$ collective diffusivity calculated from the decay of $F$ probed at $k_{c}=2 \pi / H$ for systems of $N=256(\square)$ and $N=1024(\bigcirc)$ particles. The solid line is $S^{e q}(0)^{-1}$ computed from the Carnahan-Staring approximation. The inset shows the static structure factor $S$ vs. wavenumber $(\triangleleft$ and $\triangleright)$ calculated from BD simulations (for $N=256$ particles) together with the Percus-Yevick closure (Russel et al. 1989) (dashed and solid curves).

using the time evolution of $F$. All calculations are performed in a steadily sheared suspension starting from initial random hard-sphere configurations after they have been pre-equilibrated for $\sim 10-20$ dimensionless time units depending on particle concentration and the shear rate, i.e. $P e$. Time is scaled with $a^{2} / D_{0}$ for $P e \leqslant 1$ and with $\dot{\gamma}^{-1}$ for $P e>1$. The time needed for the shear-induced microstructure to develop is conveniently monitored by the static structure factor; the typical evolution of the averaged $S(\boldsymbol{k})$ is depicted in figure 3 . Note that relaxation of $S(\boldsymbol{k})$ itself is diffusive and could be used to determine the collective diffusivity (Marchioro \& Acrivos 2001), but this approach requires one to bias the initial configurations so that $S$ is initially far from its form in the steadily sheared state.

The calculation of $F$ is performed for a range of $P e$ and volume fractions at $\boldsymbol{k}=k_{c} \boldsymbol{e}_{y, z}$ with $N=256$ particles and the same time-averaging procedure as in the previous paragraph is applied. We find for $P e>1$ that the error due to the finite box size is within the margins of the statistical error for most $\phi$. No attempt was made to simulate systems with progressively larger $N$ and extrapolate to the limit $1 / N \rightarrow 0$. The transverse collective diffusivities determined are plotted against the Péclet number for $\phi=0.15,0.25$ and 0.35 in figures $4(a), 4(b)$ and $4(c)$, respectively. The agreement with the asymptotic result (2.41) is very good, although the departure in the diffusivity from its equilibrium value $\sim S^{e q}(0)^{-1}$ occurs far beyond $P e=1$, while the asymptotic solution is formally valid for $P e<1$. Nevertheless, it seems to predict the turnover point rather accurately in all three cases.

The high- $P e$ asymptotic prediction (2.38) is in excellent agreement with the simulation results. The linear scaling of $D^{c}$ with $P e$ and the difference between $D_{y y}^{c}$ and $D_{z z}^{c}$ predicted by theory are evident in figure 4 . To demonstrate the $\phi$-dependence of the collective diffusivity we plot the high-Pe $(P e=3000)$ values of $D_{y y}^{c} / \dot{\gamma} a^{2}$ and $D_{z z}^{c} / \dot{\gamma} a^{2}$ vs. $\phi$ in a linear-log plot in figure 5. Good quantitative agreement between 


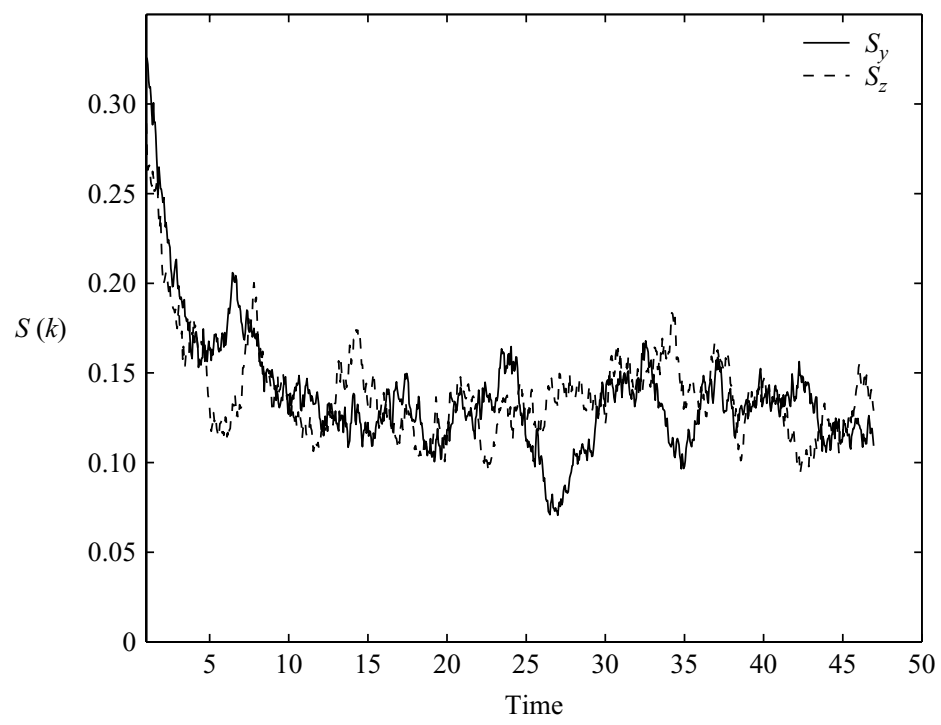

FIGURE 3. Typical evolution of the non-equilibrium static structure factor, $S(\boldsymbol{k})$, at the cutoff wave-vector $\boldsymbol{k}=2 \pi / H \boldsymbol{e}_{y, z}$, vs. dimensionless time $\dot{\gamma} t$ for $\phi=0.25$ and $P e=5$.

the asymptotic results and BD simulations is obtained without adjustable parameters. Finally, the high- $P e$ results for collective diffusivity from BD simulations are compared to existing experimental results in figure 6 . At high shear rates hydrodynamics play an important role in collective diffusion and the scaling with $\phi$ is not linear as anticipated from the dilute theory and BD simulations. Note that BD simulations of concentrated suspensions starting at $\phi \sim 0.4$ exhibit unphysical string-ordering owing to the periodicity of the boundaries and, therefore, results are presented up to $\phi=0.35$. Analogous simulations performed with accelerated Stokesian Dynamics with no Brownian motion in Leshansky \& Brady (2005) $(\triangle$ and $\nabla$ symbols in figure 6) show much better agreement with experiment than BD simulations.

\section{Static structure factor of the sheared suspension}

Finally, we address the microstructure of the sheared suspension in terms of the static structure factor at small $k$. Although we have shown that only the value of $S^{e q}(0)$ matters for the asymptotic analysis of the collective diffusivity at low $P e$, the microstructural deformation is most readily measured in light-scattering experiments via $S(\boldsymbol{k})$ (Johnson, de Kruif, \& May 1988; de Kruif et al. 1990; Wagner \& Russel 1990). Numerous investigations of the microstructure of suspensions under shear have been published (Morris \& Katyal 2002; Vermant \& Solomon 2005), but the static structure factor at zero wave-vector, $S(0)$, has not been fully addressed. Measurements at small $k$, or large wavelength, are problematic due to geometric limitations.

Dhont (1996) considered the Fourier-transformed Smoluchowski equation to study the effect of weak shear flow $(P e \ll 1)$ on the microstructure of a dilute suspension of spheres without hydrodynamic interaction. This theory anticipated no shear-induced distortion of the equilibrium microstructure in the plane orthogonal to the direction of the flow. In Bławzdziewicz \& Szamel (1993), it was first predicted theoretically that there is a non-zero deformation of the equilibrium microstructure in the plane transverse to the flow direction. They found that a dilute weakly sheared suspension of hard spheres with negligible hydrodynamic interaction undergoes shear thinning 

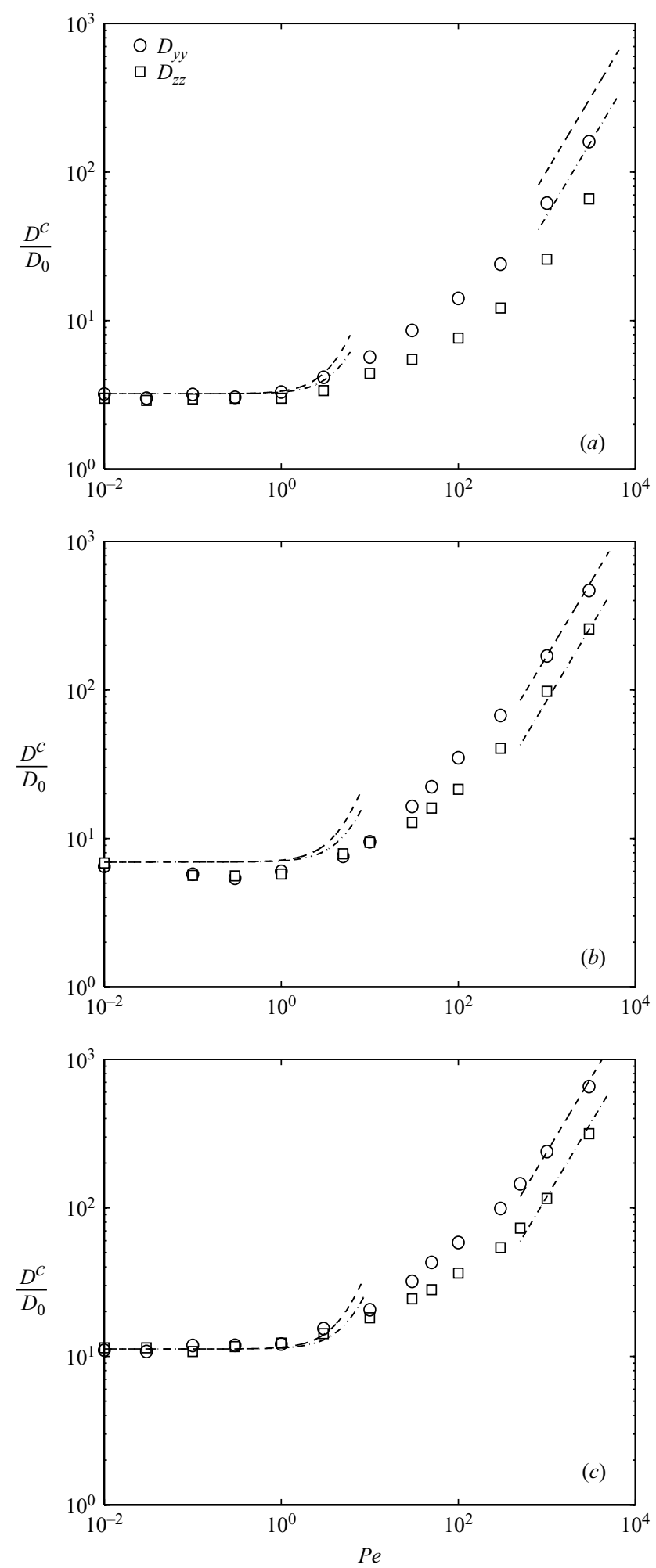

FIGURE 4. The dependence of transverse collective diffusivity on $P e$. The dashed and dashed-dotted lines represent the analytical asymptotic results for $D_{y y}^{c}$ and $D_{z z}^{c}$, respectively, for both low and high $P e$ limits. $(a) \phi=0.15 ;(b) \phi=0.25 ;(c) \phi=0.35$. 


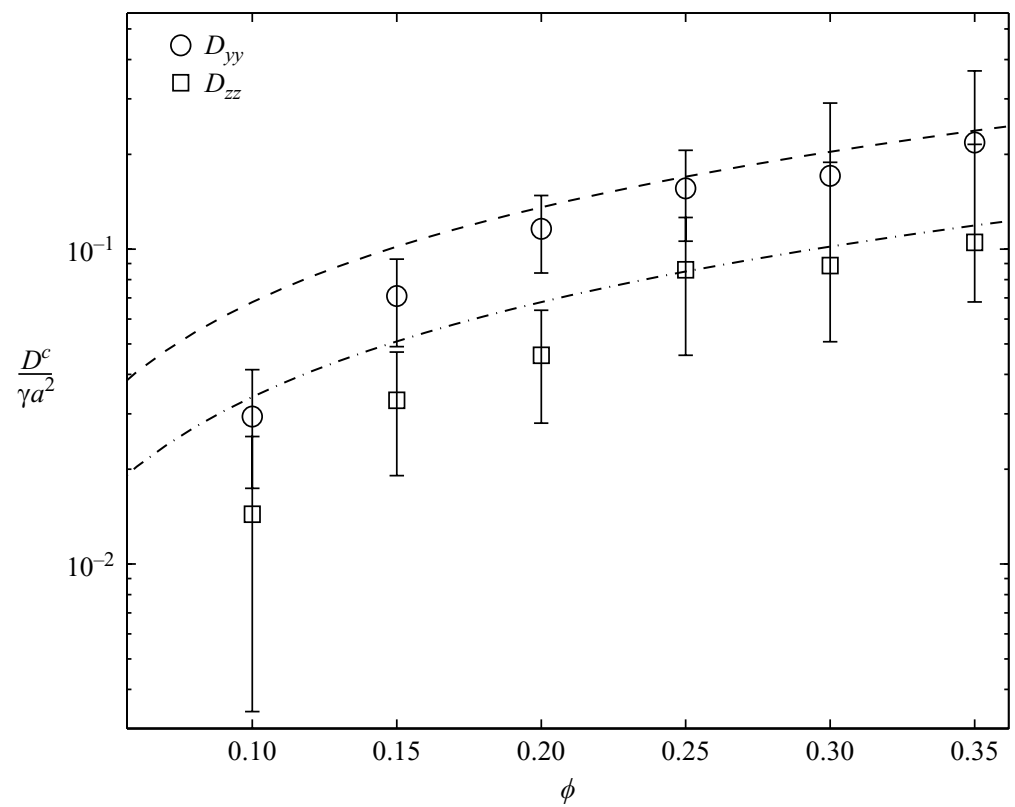

FIGURE 5. The dependence of the high-Pe values of the transverse collective diffusivity, $D_{y y}^{c} / \dot{\gamma} a^{2}(\circ)$ and $D_{z z}^{c} / \dot{\gamma} a^{2}(\square)$, on the particle volume fraction, $\phi$. The dashed and the dashed-dotted curves correspond to the asymptotic estimates (2.38) of diffusivities in the $y$ and $z$-directions, respectively.

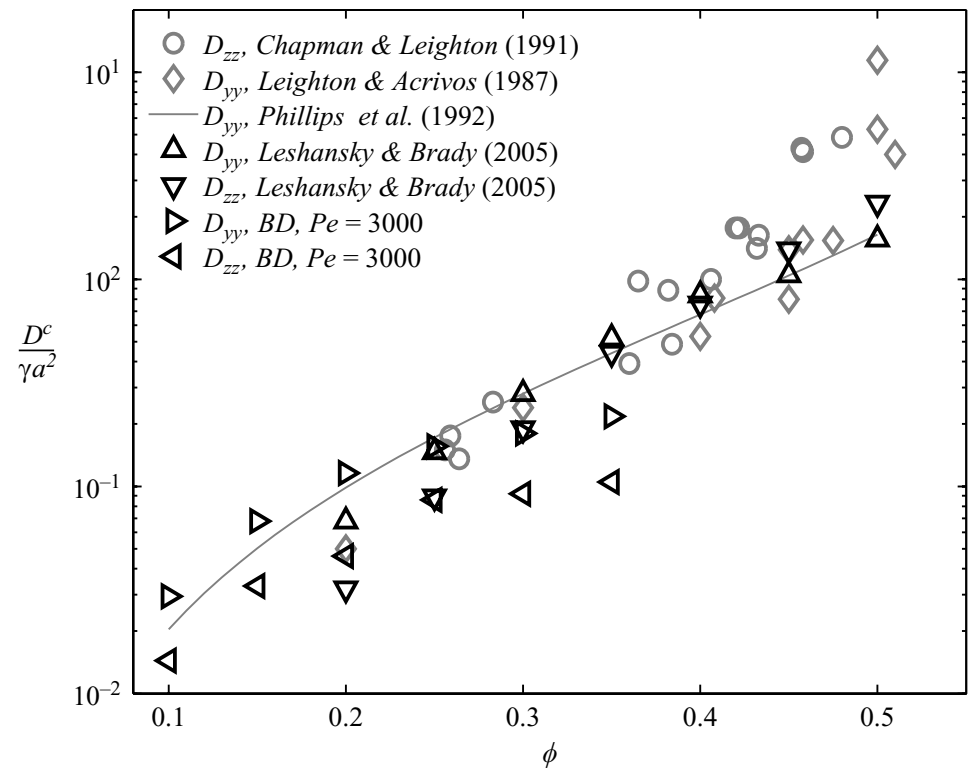

Figure 6. Transverse collective diffusivity, $D^{c} / \dot{\gamma} a^{2}$ at infinite Péclet number, as a function of particle volume fraction $\phi$ in comparison with experimental (grey symbols and the solid line) and theoretical (bold triangles) results. Accelerated Stokesian Dynamics results $(\triangle, \nabla)$ correspond to shear-induced collective diffusivity for a non-Brownian suspension (Leshansky \& Brady 2005). 


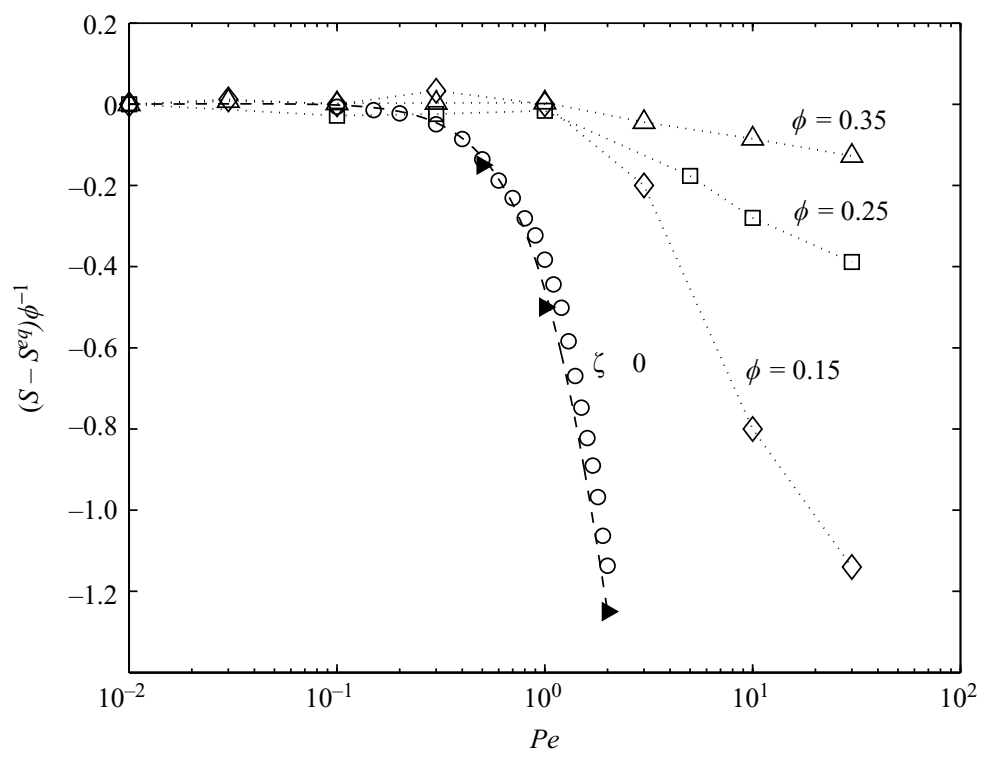

FIGURE 7. The deviation $\phi^{-1}\left[S-S^{e q}\right]$ vs. $P e . \diamond, \square, \triangle$ are the results of the BD simulations for the volume fractions $\phi=0.15,0.25$ and 0.35 , respectively; $\circ$, numerical results of Brady \& Vicic 1995); - - , the small-Pe asymptotics (A 29); $>$, numerical results of Bławzdziewicz \& Szamel (1993).

correlated with the structural changes indicated by the non-zero distortion of the static structure factor, $S(\boldsymbol{k})$, for $\boldsymbol{k}$ orthogonal to the direction of the flow. Further theoretical development by Brady \& Vicic (1995) showed that the transverse distortion of the pair-distribution function from its equilibrium value at small $P e$ scales as $P e^{2}$ with and without hydrodynamics. In neither of these studies was the non-equilibrium static structure factor, $S_{0}(P e) \equiv S(0 ; P e)$, calculated.

We compute $S(\boldsymbol{k})$ from BD simulations in two principal transverse directions, $y$ (velocity gradient) and $z$ (vorticity), after the suspension has been equilibrated for the time required to reach a steady state. The long-wavelength limit of $S(\boldsymbol{k})$ is calculated at the cutoff wavenumber, $k_{c}=2 \pi / H$, for a system of $N=256$ particles. Since the equilibrium static structure factor calculated from BD simulations is in excellent agreement with the theoretical Percus-Yevick closure for a 256-particle simulation (see the inset in figure 2), we do not attempt simulations for larger systems. The comparison between these results and the asymptotic small-Pe solution (see the Appendix) is provided in figure 7, where the deviation $\phi^{-1}\left[S_{0}\left(k_{c}, P e\right)-S_{0}^{e q}\right]$ is plotted against $P e$ for different $\phi$. Note that the dilute theory can only predict the unbounded growth of $-\Delta S_{0}$ with the increase in $P e$. Results of the $\mathrm{BD}$ simulations demonstrate that the magnitude of the flow-induced microstructural deformation diminishes as the particles concentration increases, and it remains finite as $P e \rightarrow \infty$. Indeed, as we have shown earlier in this paper, the low- $k$ deformation $\Delta S_{0}$ is correlated with the length of the wakes of low pair-probability $(g \ll 1)$ originating in the extensional quadrants. Without hydrodynamic interactions and when Brownian diffusion is entirely neglected, the pairwise theory anticipates wakes of infinite extent (Russel et al. 1989) and thus the shear-induced deformation $\Delta S_{0}$ is indefinite. When some residual Brownian transport is retained in the framework of pairwise interaction theory, the wakes are smeared 
downstream by diffusion, yielding $P e^{1 / 2}$ and $\log P e$ scaling of $\left|\phi^{-1} \Delta S_{0}\right|$ without and with hydrodynamics, respectively (for details see the Appendix).

Obviously, for non-dilute suspensions, there is an additional mechanism of the wake smearing from shear-induced gradient diffusivity, and a self-consistent theory in which this influence was included could be contemplated. It is also evident from figure 7 that the magnitude of the microstructural deformation decreases with the increase in $\phi$ in accordance with the above arguments. This is also in accord with the recent findings of Leshansky \& Brady (2005) where the shear-induced microstructural deformation $\Delta S_{0}$ calculated via accelerated Stokesian Dynamics for dense suspensions, including hydrodynamic interactions, at infinite $P e$ was found to be small.

\section{Summary and concluding remarks}

In this paper we have applied the methodology proposed in Morris \& Brady (1996) for calculation of the collective-diffusion coefficients in sheared suspensions of hard spheres. The Fourier-transform method is applied to identify the form of the collective diffusivity from the time evolution of the density fluctuation autocorrelation for an arbitrary unbounded linear flow.

We found that for weakly sheared suspensions $(P e<1)$, the collective diffusivity possesses a regular asymptotic expansion in integer powers of $P e$ up to $O\left(\mathrm{Pe}^{2}\right)$, while for the self-diffusivity (Morris \& Brady 1996) there is already a singularity at $O\left(\mathrm{Pe}^{3 / 2}\right)$. As for the self-diffusion coefficient, the leading shear-induced contribution to the collective diffusivity is linear in the imposed flow, and scales as Pe. For instance, in a simple shear flow, $\dot{\Gamma}_{i j}=\delta_{1 i} \delta_{2 j}$, this contributes to the off-diagonal component, $D_{12}^{c}$. As found for the self-diffusivity, this off-diagonal diffusivity is negative.

The diagonal components of the collective diffusivity in the directions of velocity gradient and vorticity are quadratic in the applied flow and only weakly affected by the magnitude of the applied shear, while the deviation from an equilibrium value scales as $P e^{2}$ at small $P e$. This weak dependence has been confirmed by BD simulations, showing very good agreement with derived small-Pe asymptotic expressions up to $P e \sim O(1)$. Hydrodynamic interactions result in an enhanced transverse collective diffusivity as the coefficients of terms proportional to $\hat{\boldsymbol{E}} \cdot \hat{\boldsymbol{E}}$ and $(\hat{\boldsymbol{E}}: \hat{\boldsymbol{E}}) \boldsymbol{I}$ in $(2.67)$ are larger than the similar terms in (2.41). The major contribution to these terms arises from the velocity fluctuation integral (2.64), which is zero when hydrodynamic interactions are neglected. In contrast to the macroscopically quiescent suspension, where hydrodynamics hinders collective diffusion at $O(\phi)$, hydrodynamic interactions among particles in a linear bulk flow augment collective diffusion. The antisymmetric contribution $(\propto \hat{\boldsymbol{E}} \cdot \hat{\boldsymbol{\Omega}})$ is small if hydrodynamics is considered, resulting in a higher diffusivity in the velocity-gradient direction relative to that in vorticity direction.

The hydrodynamic interactions between suspended particles do not affect the scaling of the transverse collective-diffusion components. The leading-order contribution at $O(P e)$, on the other hand, is complicated due to the long-range nature of the particle relative velocity. Renormalization of the divergent integral leads to abnormal scaling for the cross collective-diffusion component, $\sim \log (a k)$, yielding a weak blow-up. The question of whether this abnormal diffusivity is of a physical origin is unclear. Without making any statements, we would like to point out an analogous divergence issue in sedimenting suspensions, where the variance in the sedimentation speed for a homogeneous suspension of solid spheres is predicted to 
grow without bound in the infinite particle number limit, $\left\langle U^{2}\right\rangle \sim N^{1 / 3} \phi^{2 / 3}$ (Caflisch \& Luke 1985), or in our terms $\sim \phi(a k)^{-1}$ since $k \propto(\phi / N)^{1 / 3}$ as $k \rightarrow 0$. The weak logarithmic blow-up in our case for the collective diffusivity $\sim \log (a k)$ vs. the $(a k)^{-1}$ singularity in sedimentation is probably a consequence of weaker hydrodynamic interactions between neutrally buoyant particles in linear flows, $\left|\boldsymbol{U}_{12}\right| \sim|\boldsymbol{r}|^{-2}$ vs. $|\boldsymbol{r}|^{-1}$ in sedimentation.

For strongly sheared suspensions, the diffusivity scales with the shear rate, $\boldsymbol{D}^{c} \sim \dot{\gamma} a^{2}$, and we found that $\boldsymbol{D}^{c}=6 \boldsymbol{D}_{\infty}^{s}$. The results of the BD simulations validate unequivocally not only the linear scaling of $\boldsymbol{D}^{c}$ with $P e$, but also show very good quantitative agreement with the asymptotic results even for moderate volume fractions (see figure 5). This result could be anticipated, as three-particle effects are only involved through hydrodynamics (see (2.27)) and the pairwise theory is expected to give accurate estimates of the collective-diffusivity beyond the dilute limit.

Finally, we have calculated the static structure factor of a dilute suspension in the simple shear flow. Although we have shown that shear-induced deformation of the microstructure does not affect the outcome of the asymptotic analysis for collective diffusivity, the static structure factor $S(\boldsymbol{k})$ is one of the most intensively studied properties in the colloidal literature and it can be readily measured in small-angle neutron scattering or dynamic light scattering experiments (see Vermant \& Solomon 2005 and references therein). The method of matched asymptotic expansions was applied to construct the uniformly valid expansion for $g(\boldsymbol{r})$ in the small-Pe limit and to compute $S(0)$. This theory is in excellent agreement with previously reported numerical results (Bławzdziewicz \& Szamel 1993) and with the results obtained using the numerical approach of Vicic (1999). When hydrodynamic interactions between particles are neglected, the microstructural deformation $\left(S_{0}(P e)-S_{0}^{e q}\right) \phi^{-1}$ was shown to be negative and diminishing with the rate $\sim \sqrt{P e}$ for $P e \gg 1$. On the other hand, when hydrodynamic interactions are included, we found that $\phi^{-1} \Delta S$ is positive up to $P e \approx 1.5$, and diminishes with the rate $\sim \log P e$ at large Pe. The BD simulations show that $\phi^{-1} \Delta S(0)$ is a decreasing function of $P e$ as found from the pairwise $O(\phi)$-theory, while the magnitude of the microstructural deformation reduces with the growth of $\phi$, as the additional 'stirring' mechanism due to shear-induced diffusion is operable in concentrated suspensions.

The authors thank Johan Bergenholtz for the help with calculating the microstructural deformation of a dilute suspension at large Péclet numbers. A.M.L and J.F.B. acknowledge the support of the Binational Science Foundation (BSF) through grant \#890020.

\section{Appendix. Microstructure of dilute suspension under shear}

As shown in Brady \& Vicic (1995), the perturbation to the pair-distribution function, $p(\boldsymbol{r})$ defined by $g(\boldsymbol{r})=g^{e q}(r)[1+p(\boldsymbol{r})]$ is governed in the dilute limit by

$$
\nabla_{r} \cdot \boldsymbol{D}_{r} \cdot \nabla_{r} p=P e \nabla_{r} \cdot \boldsymbol{U}_{r}(1+p), \quad|\boldsymbol{r}|>2,
$$

subject to the boundary conditions

$$
\begin{aligned}
\boldsymbol{n} \cdot \boldsymbol{D}_{r} \cdot \nabla_{r} p & =P e \boldsymbol{n} \cdot \boldsymbol{U}_{r}(1+p) \text { at } \quad r=2, \\
p & \sim 0 \text { as } \quad r \rightarrow \infty .
\end{aligned}
$$


Here, $\boldsymbol{U}_{r}=\boldsymbol{U}_{2}-\boldsymbol{U}_{1}$ is the relative velocity of two particles arising from the imposed shearing flow and $\boldsymbol{D}_{r}$ is the relative diffusivity, given by

$$
\begin{aligned}
& \boldsymbol{U}_{r}=(\hat{\boldsymbol{E}}+\hat{\boldsymbol{\Omega}}) \cdot \boldsymbol{r}-\boldsymbol{r} \cdot \hat{\boldsymbol{E}} \cdot[A(r) \boldsymbol{n n}+B(r)(\boldsymbol{I}-\boldsymbol{n} \boldsymbol{n})], \\
& \boldsymbol{D}_{r}=2[G(r) \boldsymbol{n} \boldsymbol{n}+H(r)(\boldsymbol{I}-\boldsymbol{n n})]:
\end{aligned}
$$

respectively. It can be readily seen that that for small $P e$ the problem is singularly perturbed, i.e. at distances $r \sim P e^{-1 / 2}$ the convection is as important as diffusion in (A 1). It has been demonstrated in Brady \& Vicic (1995) that for moderate distances, $r \ll O\left(P e^{-1 / 2}\right)$, the solution of (A 1)-(A 3) can be written as a regular asymptotic expansion in powers of $P e$ up to $O\left(P e^{5 / 2}\right)$,

$$
p(\boldsymbol{r})=P e p_{1}(\boldsymbol{r})+P e^{2} p_{2}(\boldsymbol{r})+O\left(P e^{5 / 2}\right),
$$

with $p_{1}$ and $p_{2}$ given by

$$
\begin{gathered}
p_{1}=-h_{1}(r) \boldsymbol{n} \cdot \hat{\boldsymbol{E}} \cdot \boldsymbol{n}, \\
p_{2}=-\left[h_{2}(r)(\boldsymbol{n} \cdot \hat{\boldsymbol{E}} \cdot \boldsymbol{n})^{2}+h_{3}(r) \boldsymbol{n} \cdot \hat{\boldsymbol{E}} \cdot \hat{\boldsymbol{\Omega}} \cdot \boldsymbol{n}+h_{4}(r) \boldsymbol{n} \cdot \hat{\boldsymbol{E}} \cdot \hat{\boldsymbol{E}} \cdot \boldsymbol{n}+h_{5}(r) \hat{\boldsymbol{E}}: \hat{\boldsymbol{E}}\right],
\end{gathered}
$$

where $\hat{\boldsymbol{\Omega}}$ is the vorticity tensor of the bulk flow, and $\boldsymbol{n}=\boldsymbol{r} / r$. Making use of (A 4-A 5) and substituting (A 7)-(A 8) into (A 1)-(A 3) results in a system of coupled ODEs $\dagger$ for $h_{i}$,

$$
\begin{aligned}
& \mathrm{L}_{2} h_{1}(r)=-W(r), \\
& \mathrm{L}_{4} h_{2}(r)=\left[\frac{1}{2} W(r)-1+B(r)\right] h_{1}(r)+\frac{1}{2} r[1-A(r)] h_{1}^{\prime}(r), \\
& \mathrm{L}_{2} h_{3}(r)=h_{1}(r), \\
& \mathrm{L}_{2} h_{4}(r)=-8 \frac{H(r)}{r^{2}} h_{2}(r)+[1-B(r)] h_{1}(r), \\
& \mathrm{L}_{0} h_{5}(r)=-2 \frac{H(r)}{r^{2}} h_{4}(r) .
\end{aligned}
$$

The operator $\mathrm{L}_{\alpha}$ is defined by

$$
\mathrm{L}_{\alpha}=\frac{1}{r^{2}} \frac{\mathrm{d}}{\mathrm{d} r}\left[r^{2} G(r) \frac{\mathrm{d}}{\mathrm{d} r}\right]-\alpha(\alpha+1) \frac{H(r)}{r^{2}} .
$$

and $W(r)=3[B(r)-A(r)]-r A^{\prime}(r)$. All $h_{i}$ decay at large $r$ while the inner boundary conditions (A 2) at $r=2$ become

$$
\begin{aligned}
& G(r) h_{1}^{\prime}(r)=-[1-A(r)], \\
& G(r) h_{2}^{\prime}(r)=h_{1}[1-A(r)], \\
& G(r) h_{i}^{\prime}(r)=0, \quad i=3,4,5,
\end{aligned}
$$

Here $A(r), B(r), G(r)$ and $H(r)$ are non-dimensional pair hydrodynamic functions (Kim \& Karrila 1991).

\section{Without hydrodynamic interactions}

In the absence of hydrodynamic interactions, (A 1)-(A 3) reduce to

$$
\begin{aligned}
2 \nabla_{r}^{2} p & =P e \dot{\boldsymbol{\Gamma}} \cdot \boldsymbol{r} \cdot \nabla_{r} p, \quad r>2, \\
\boldsymbol{n} \cdot \nabla_{r} p & =P e \boldsymbol{n} \cdot \hat{\boldsymbol{E}} \cdot \boldsymbol{n}(1+p) \text { at } \quad r=2, \\
p & \sim 0 \text { as } \quad r \rightarrow \infty .
\end{aligned}
$$

$\dagger$ The original system of ODEs for $h_{1}-h_{5}$ ((A3)-(A7) in the Appendix of Brady \& Vicic 1995) contains a number of errors, and, therefore, we have chosen to re-calculate the functions $h_{i}$ here. 
Under these conditions, the functions $h_{i}$ in (A 7)-(A 8) can be found in closed form as the solution of (A 9)-(A 16) with $A(r)=B(r)=0$ and $G(r)=H(r)=1$ (Brady \& Vicic 1995):

$$
\left.\begin{array}{ll}
h_{1}(r)=\frac{16}{3} \frac{1}{r^{3}}, & h_{1}(2)=\frac{2}{3}, \\
h_{2}(r)=\frac{2}{3}\left(\frac{1}{r}-\frac{16}{r^{5}}\right), & h_{2}(2)=0, \\
h_{3}(r)=-\frac{8}{27}\left(\frac{3}{r}-\frac{4}{r^{3}}\right), & h_{3}(2)=-\frac{8}{27}, \\
h_{4}(r)=-\frac{32}{63}\left(\frac{5}{r^{3}}-\frac{12}{r^{5}}\right), & h_{4}(2)=-\frac{8}{63}, \\
h_{5}(r)=-\frac{4}{945}\left(\frac{105}{r}-\frac{200}{r^{3}}+\frac{144}{r^{5}}\right), & h_{5}(2)=-\frac{128}{945} .
\end{array}\right\}
$$

One cannot calculate flow-induced deformation to the microstructure in (2.40) using this regular expansion, because it results in conditionally convergent or divergent integrals due to slow decay of $h_{1}\left(\sim r^{-3}\right)$ and $h_{2}-h_{5}\left(\sim r^{-1}\right)$. Instead, one must construct a uniformly valid asymptotic expansion of $p$, where the slowly decaying terms will be matched to the solution in the 'outer' region, $r \sim O\left(P e^{-1 / 2}\right)$. We introduce an outer expansion $\mathscr{P}(\rho)=\sum_{n} H_{n}(\varepsilon) \mathscr{P}_{n}$ where $\boldsymbol{\rho}=\left(X_{1}, X_{2}, X_{3}\right)=\varepsilon \boldsymbol{r}=O(1)$ is the scaled outer variable with $\varepsilon \equiv P e^{1 / 2}$. It follows from (A 1 ) that any term of the outer expansion should satisfy

$$
2 \nabla_{\rho}^{2} \mathscr{P}_{n}=\dot{\boldsymbol{\Gamma}} \cdot \boldsymbol{\rho} \cdot \nabla_{\rho} \mathscr{P}_{n}, \quad \text { and } \quad \mathscr{P}_{n} \rightarrow 0 \quad \text { as } \quad \rho \rightarrow \infty .
$$

Next we construct solutions $\mathscr{P}_{n}$ for simple shear flow, $\dot{\Gamma}_{i j}=\delta_{1 i} \delta_{2 j}$ as a superposition of solutions $\mathscr{T}_{\alpha \beta}$ corresponding to multipole sources $\left(\partial / \partial x_{1}\right)^{\alpha}\left(\partial / \partial x_{2}\right)^{\beta} \delta(\boldsymbol{r})$,

$$
\mathscr{P}_{n}=\sum_{\alpha, \beta=0}^{\infty} C_{n}^{\alpha \beta} \mathscr{T}_{\alpha \beta}(\boldsymbol{\rho}),
$$

where the $\mathscr{T}_{\alpha \beta}$ can be obtained from the fundamental solution of (A 21) found by Elrick (1962),

$$
\mathscr{T}_{\alpha \beta}(\rho)=\int_{0}^{\infty} \frac{\mathrm{d} s}{\left(1+s^{2} / 3\right) s^{3 / 2}}\left(\frac{\partial}{\partial X_{1}}\right)^{\alpha}\left(\frac{\partial}{\partial X_{2}}+2 s \frac{\partial}{\partial X_{1}}\right)^{\beta} \mathrm{e}^{-\rho^{2} / 16 s} \mathrm{e}^{\mathscr{E}},
$$

where

$$
\mathscr{E}=\frac{s\left(X_{1}^{2}-3 X_{2}^{2}\right)+6 X_{1} X_{2}}{48\left(1+s^{2} / 3\right)} .
$$

The expansion coefficients $H_{n}(\varepsilon)$ and $C_{n}^{\alpha \beta}$ are determined by matching to the inner solution (A 6)-(A 8) as $|\boldsymbol{\rho}| \rightarrow 0$. Note that the solution to the full problem (A 1)(A 3) for an arbitrary $P e$ can be constructed numerically via (A 23) (with $H_{n}=1$ ). In this case, the coefficients $C^{\alpha \beta}$ are to be determined by the boundary conditions (A 2) directly, yielding an infinite linear system of equations for the coefficients $C^{\alpha \beta}$ (Bławzdziewicz \& Szamel 1993).

The first approximation of the outer expansion $\mathscr{P}$ should match the quadrupole forcing $p_{1}$ (A 7) re-written in the outer variables, i.e.

$$
\mathscr{P}(\boldsymbol{\rho}) \rightarrow P e^{5 / 2}\left(-\frac{16}{3} \frac{\boldsymbol{n} \cdot \hat{\boldsymbol{E}} \cdot \boldsymbol{n}}{\rho^{3}}+O\left(\rho^{-1}\right)\right),
$$


where $O\left(P e^{5 / 2} \rho^{-1}\right)$ terms are generated from $r^{-1}$ terms in the second-order perturbation $p_{2}$ (A 8) $\left(h_{2}, h_{3}\right.$ and $\left.h_{5}\right)$. This matching condition leads to $H_{1}=\varepsilon^{5}$. The choice $\alpha=\beta=1$ with $C_{1}^{11}=4 / 9 \sqrt{\pi}, C_{1}^{\alpha \beta}=0$ for $\alpha, \beta \neq 1$ provides the required solution satisfying (A 24) as $\rho \rightarrow 0$,

$$
\mathscr{P}_{1}=-\frac{4}{9 \sqrt{\pi}} \mathscr{T}_{11} .
$$

One can show (through some lengthy algebra) that the asymptotic form of $\mathscr{P}_{1}$ as $\rho \rightarrow 0$ is given by

$$
\mathscr{P}_{1} \sim-\frac{16}{3} \frac{X_{1} X_{2}}{\rho^{5}}+\frac{2}{9} \frac{X_{2}^{2}-X_{1}^{2}}{\rho^{3}}-\frac{2}{3} \frac{X_{1}^{2} X_{2}^{2}}{\rho^{5}}+\frac{2}{9 \rho}+\mathscr{A}+O(\rho) .
$$

Rewritten in inner variables, $\mathscr{P}_{1}$ can be shown to match $p_{1}$ (A 7) and the $r^{-1}$ terms in $p_{2}$ (A 8)

$$
p \sim-P e h_{1}(r) \boldsymbol{n} \cdot \hat{\boldsymbol{E}} \cdot \boldsymbol{n}+P e^{2}\left(-\frac{2}{3} \frac{(\boldsymbol{n} \cdot \hat{\boldsymbol{E}} \cdot \boldsymbol{n})^{2}}{r}+\frac{8}{9} \frac{\boldsymbol{n} \cdot \hat{\boldsymbol{E}} \cdot \hat{\boldsymbol{\Omega}} \cdot \boldsymbol{n}}{r}+\frac{4}{9} \frac{\hat{\boldsymbol{E}}: \hat{\boldsymbol{E}}}{r}\right)+\mathscr{A} P e^{5 / 2},
$$

where $\mathscr{A}=\left[\Gamma\left(-\frac{1}{4}\right) \Gamma\left(\frac{11}{4}\right)+\Gamma\left(\frac{3}{4}\right) \Gamma\left(\frac{7}{4}\right)\right] / 3^{13 / 4} \pi$.

Matching the next two terms of the outer expansion with the $r^{-3}$ and $r^{-5}$ terms of $p_{2}$ yields $H_{2}=\varepsilon^{7}$ and $H_{3}=\varepsilon^{9}$. Omitting the details of the matching procedure, these terms are found as

$$
\mathscr{P}_{2}=\frac{4}{63 \sqrt{\pi}}\left(\frac{11}{9} \mathscr{T}_{20}+\frac{4}{9} \mathscr{T}_{02}\right), \quad \mathscr{P}_{3}=\frac{8}{315 \sqrt{\pi}} \mathscr{T}_{22} .
$$

Note that, because $h_{2}-h_{5}$ contain only terms with odd powers of $1 / r$, the outer expansion involves only terms proportional to $P e^{2 n+1 / 2}$. Therefore, in the limit $\rho \rightarrow 0$ the three-term expansion (A 25)-(A 28) matches the two-term inner expansion (A 6) exactly, and it represents a uniformly valid solution of the full problem (A 1)-(A 3) up to $O\left(P e^{5 / 2}\right)$ for small $P e$. Interestingly, in Bławzdziewicz \& Szamel (1993) the distortion of the pair-distribution function $g(\boldsymbol{r})$ for an arbitrary $P e$ was determined by solving for the coefficients of the multipole expansion $C^{\alpha \beta}$ numerically, with the finding that for $P e \leqslant 2$ the truncation level $l=\alpha+\beta \leqslant 4$ is sufficient. Our theory provides the asymptotic form of these coefficients at small $P e$, indicating that only multipoles with even $l$ contribute, i.e. $\mathscr{T}_{11}, \mathscr{T}_{20}$, etc.

To obtain the non-equilibrium static structure factor (2.40) we perform a numerical integration of the constructed solution in the whole space for $r>2$. In figure 8 the results of the numerical integration of the leading term $P e^{5 / 2} \mathscr{P}_{1}$ are presented ( $\square$ symbols) on a log-linear plot. The inset shows the calculated values of $S_{0}$ near $P e=0$. Although the angular integration over the quadrupole forcing term in the inner expansion (A 7) yields zero, an $O(P e)$ contribution to $S_{0}$ could be anticipated from the first unmatched constant term $\mathscr{A} P e^{5 / 2}$ in (A 27). An $O\left(P e^{2}\right)$ contribution to $S_{0}$ is expected from $r^{-1}$ terms in (A 7), while the contribution of the outer region is restricted to $O\left(P e^{5 / 2}\right)$. Thus, the best fit of the results of numerical integration of $P e^{5 / 2} \mathscr{P}_{1}$ to the polynomial form

$$
\left(S_{0}-S_{0}^{e q}\right) \phi^{-1} \simeq \frac{3}{4 \pi}\left(c_{0} P e+c_{1} P e^{2}+c_{2} P e^{5 / 2}\right)
$$

gives $c_{0}=0.254, c_{1}=-3.77$, and $c_{2}=1.54$. 


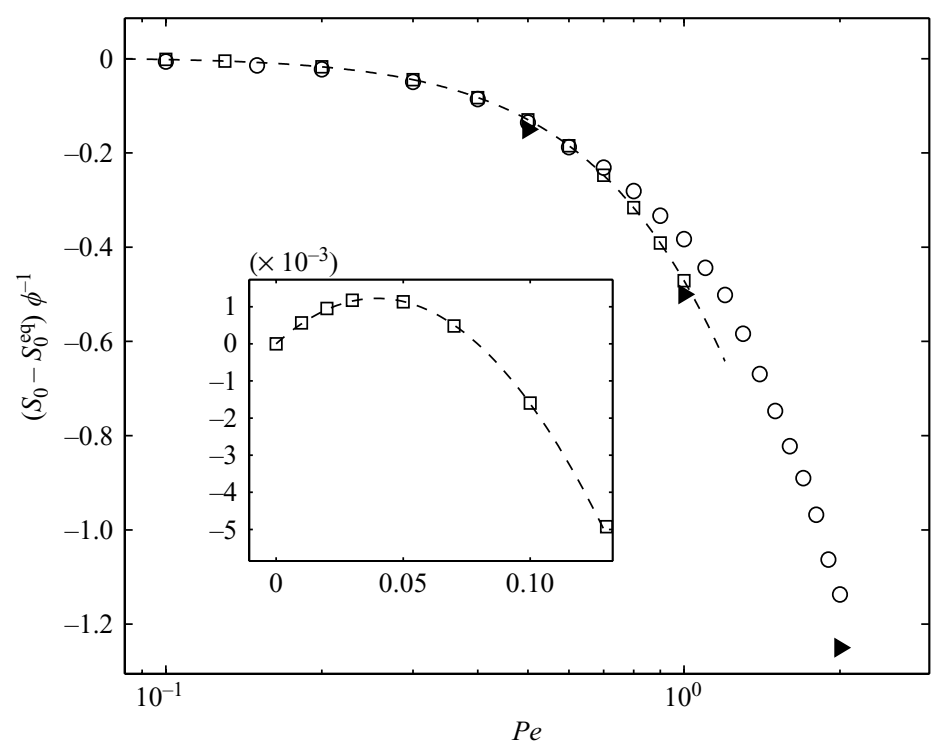

FIGURE 8. Distortion of the steady static structure factor $\left[S_{0}(P e)-S_{0}^{e q}\right] / \phi$ from its the equilibrium form at zero wave-vector, $k=0$ : $\bigcirc$, spherical harmonic expansion; $\square$, results of the numerical integration of a one-term asymptotic expansion, $P e^{5 / 2} \mathscr{P}_{1} ;---$, the best fit (A 29); numerical results of Bławzdziewicz \& Szamel (1993). The inset shows the calculated asymptotic behaviour near $P e=0$.

Further, since the angular integration over $r^{-3}$ and $r^{-5}$ terms in $p_{2}$ (A 8) yields zero as well, it is anticipated that the contribution from (A 28) is restricted to $O\left(P e^{7 / 2}\right)$. Although matching $\mathscr{P}_{2}$ results in a constant term $\mathscr{B} P e^{7 / 2}$ in the inner expansion, suggesting that there should be an $O\left(P e^{2}\right)$ contribution to $S_{0}$, the integral of $P e^{7 / 2} \mathscr{P}_{2}$ scales as $P e^{7 / 2}$, having already started at $P e \sim 0.01$ (not shown), suggesting that this contribution is very small. Thus, one can compute $S_{0}$ in the first approximation up to $O\left(P e^{5 / 2}\right.$ ) from $P e^{5 / 2} \mathscr{P}_{1}$ (A 25) alone. The asymptotic result (A 29) (dashed line in figure 8 ) is in very good agreement with numerical calculations of Bławzdziewicz \& Szamel (1993). We calculate the distortion $\left[S_{0}-S_{0}^{e q}\right] / \phi$ using a different approach developed by Vicic (1999), where $p(\boldsymbol{r})$ is expanded in spherical harmonics as

$$
p(\boldsymbol{r}, P e)=\sum_{l=0}^{L} \sum_{m=-l}^{m=l} B_{l m}(r, P e) Y_{l}^{m}(\theta, \varphi),
$$

and the radial functions $B_{l m}$ are determined numerically from the infinite set of coupled ODEs (Vicic 1999). From orthogonality of the surface spherical harmonics $Y_{l}^{m}$ it follows that only the first term in the expansion contributes, and the distortion of the static structure from equilibrium is determined as

$$
\phi^{-1} \Delta S_{0}=\frac{3}{2 \sqrt{\pi}} \int_{r=2}^{\infty} B_{00}(r, P e) r^{2} \mathrm{~d} r .
$$

The results of the numerical integration in (A 31) with $L \leqslant 16$ for small $P e$ are presented in figure 8 (० symbols). Agreement between the full numerical solution and the asymptotic result (A 29) is excellent up to $P e \simeq 0.6$. The close agreement between the numerical results of Bławzdziewicz \& Szamel (1993) and the asymptotic solution up to $P e \sim 1$ shows that the truncation level $L=2$ in multipole expansion is sufficient. 


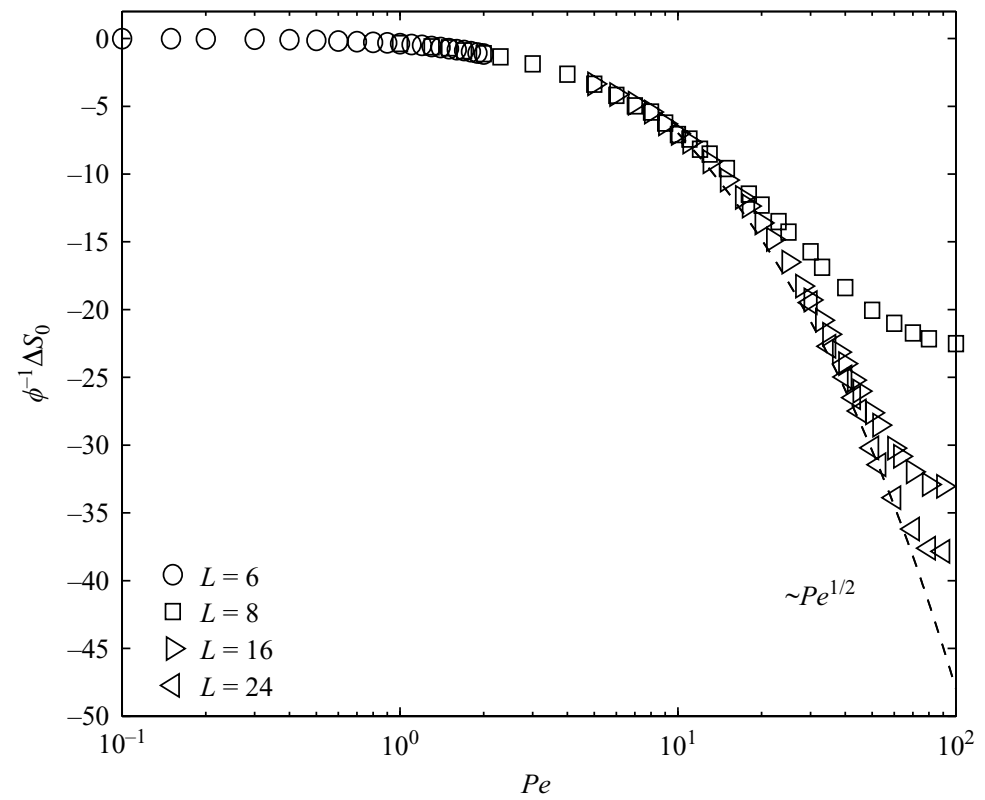

Figure 9. Distortion $\phi^{-1} \Delta S_{0}$ vs. $P e$ calculated using expansion (A 31) for different truncation levels $L$. The dashed line corresponds to an anticipated high-Pe asymptote $\sim P e^{1 / 2}$.

The distortion of the static structure factor for moderate $P e$, calculated from (A 31) upon increasing the truncation level to $L=24$ is depicted in figure 9 together with the anticipated high- $P e$ asymptote, $-\phi^{-1} \Delta S_{0} \sim P e^{1 / 2}$ (dashed line).

Pairwise hydrodynamic interactions

When pairwise hydrodynamic interactions between particles are included, the equations governing the perturbation functions $h_{i}$ (A9)-(A 13) must be solved numerically. The inner boundary conditions in this case are the same for all $h_{i}$ :

$$
G(r) h_{i}^{\prime}(r)=0 \quad \text { at } \quad r=2 .
$$

The solution for $p_{1}$ was first found in Batchelor (1977), and $h_{2}-h_{5}$ have been determined numerically in Brady \& Vicic (1995). We recalculate $h_{i}$ using a 'shooting' method: near-field asymptotic results are exploited to integrate outward where the solutions are matched to the far-field asymptotic expansion at some large $L$. More precise pairwise hydrodynamic functions are used here than in prior work, resulting in a more accurate numerical solution for $h_{i}$. The matched far-field solutions to $h_{1}-h_{5}$ are given by

$$
\begin{gathered}
h_{1} \sim \frac{\alpha_{1}}{r^{3}}+\frac{3 \alpha_{1}-25}{r^{4}}, \quad \alpha_{1}=8.904, \\
h_{2} \sim \frac{33 \alpha_{1}-200}{96 r^{2}}+\frac{\alpha_{1}\left(5 r^{4}-16\right)}{40 r^{5}}, \\
h_{3} \sim-\frac{\alpha_{1}}{6 r}-\frac{4 \alpha_{1}-25}{8 r^{2}}-\frac{\alpha_{3}}{r^{3}}, \quad \alpha_{3}=0.608 \\
h_{4} \sim-\frac{3 \alpha_{1}-25}{24 r^{2}}-\frac{\alpha_{4}}{r^{3}}, \quad \alpha_{4}=3.703, \\
h_{5} \sim-\frac{3 \alpha_{1}-25-18 \alpha_{5}}{24 r^{2}}-\frac{\alpha_{5}}{r}, \quad \alpha_{5}=0.736 .
\end{gathered}
$$




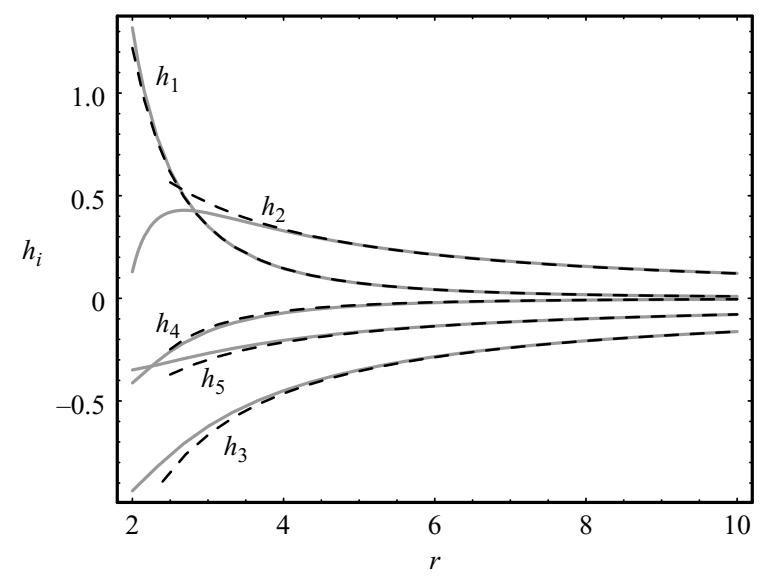

FIGURE 10. Functions $h_{1}-h_{5}$ for pairwise hydrodynamic interactions vs. the separation distance $r$; ---, asymptotic solutions (A 32)-(A 36);

Fortunately, the far-field asymptotic solutions converge to $h_{i}$ at $r \simeq 4$, and moderate $L \sim 10$ can thus be used for matching. The calculated functions $h_{i}$ (solid curves) together with their far-field asymptotic solutions (A 32)-(A 36) (dashed curves) are plotted in figure 10 .

Concerning the microstructural deformation, $\Delta S_{0}$, we face the same problem as when hydrodynamic interactions were neglected, i.e. the 'inner' solution does not allow integration of $p(\boldsymbol{r})$ due to the slow decay of $h_{i}$ at large $r$. The construction of the uniformly valid solution is more difficult with hydrodynamic interactions, and only the leading term of the outer expansion can be derived in a simple fashion. The first approximation to the solution for the pair-distribution function $p_{1}$ far from contact is again the quadrupole term (up to a multiplicative constant), as without interactions, (A 7), i.e.

$$
p_{1}(\boldsymbol{r})=-q(r) \boldsymbol{n} \cdot \hat{\boldsymbol{E}} \cdot \boldsymbol{n} \sim-\frac{\alpha_{1}}{r^{3}} \boldsymbol{n} \cdot \hat{\boldsymbol{E}} \cdot \boldsymbol{n}+O\left(r^{-4}\right) \quad \text { as } \quad r \rightarrow \infty .
$$

At distances $r \sim O\left(P e^{-1 / 2}\right)$, the relative diffusivity $\boldsymbol{D}_{r} \sim 2 \boldsymbol{I}+O\left(r^{-1}\right)$ and the relative velocity $\boldsymbol{U}_{r} \sim \dot{\boldsymbol{\Gamma}} \cdot \boldsymbol{r}+O\left(r^{-3}\right)$ and, thus, the pair-distribution function, to the first approximation, is governed by the same equation as without hydrodynamics (A 21), $\mathscr{P} \approx \varepsilon^{5} \mathscr{P}_{1}$, where $\mathscr{P}_{1}$ has the same form as (A 25 ) (up to a multiplicative constant),

$$
\mathscr{P}_{1}=-\frac{\alpha_{1}}{12 \sqrt{\pi}} \mathscr{T}_{11} .
$$

It is readily seen that (A 38) when rewritten in inner spatial variables in the limit of $\rho \rightarrow 0$ matches the $O\left(r^{-3}\right)$ quadrupole term of $h_{1}$ (A 32) and the $O\left(r^{-1}\right)$ terms in the far-field inner expansion of $h_{2}, h_{3}$ and $h_{5}$ (A 33)-(A 36) in the same way as in (A 26)-(A 27). Unfortunately, the one-term outer expansion is not a complete solution to the problem at $O(P e)$, as was the case without hydrodynamic interactions: it does not match $p_{1}$ exactly. The unmatched $O\left(r^{-4}\right)$ terms in $p_{1}$ may contribute to $\Delta S_{0}$ at $O(P e)$ and, therefore, we do not expect to arrive at the asymptotically correct result by integrating the one-term outer expansion, $\varepsilon^{5} \mathscr{P}_{1}$. The derivation of the next term in the outer expansion, $\varepsilon^{6} \mathscr{P}_{2}$, matching the $O\left(r^{-4}\right)$ term of the far-field inner solution 


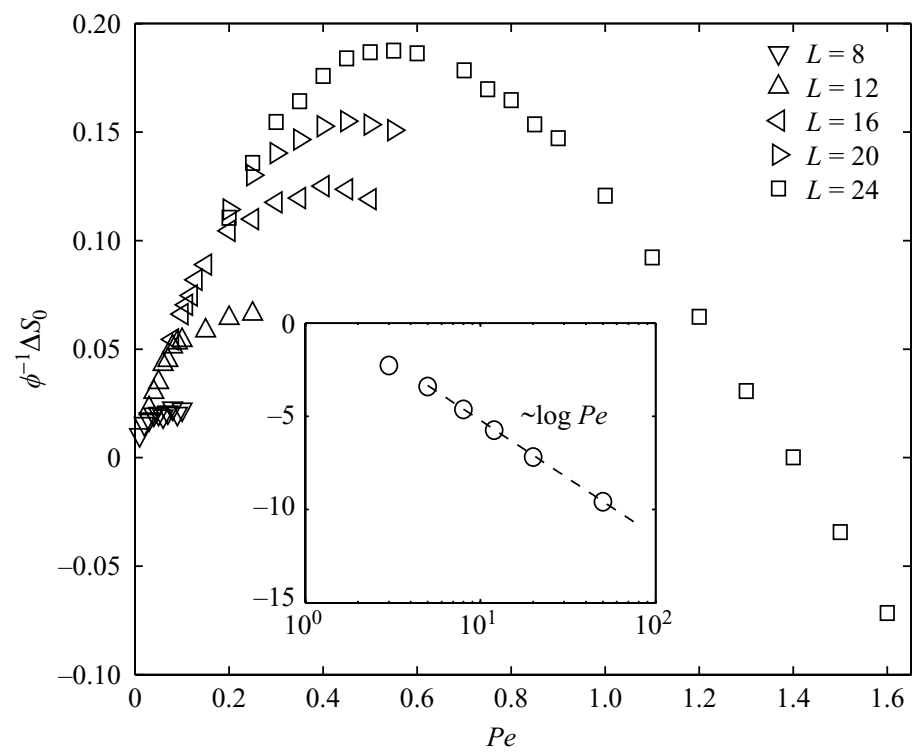

FIGURE 11. Microstructural deformation, $\phi^{-1} \Delta S_{0}$, calculated for pairwise hydrodynamics as a function of the Péclet number upon varying the truncation level $L$. The inset shows the large-Pe numerical results obtained via the finite-difference scheme of Bergenholtz et al. (2002).

for $p_{1}$ (A 32), requires the solution of

$$
2 \nabla_{\rho}^{2} \mathscr{P}_{2}-\dot{\boldsymbol{\Gamma}} \cdot \boldsymbol{\rho} \cdot \nabla_{\rho} \mathscr{P}_{2}=\frac{3}{2} \rho^{-1}\left(\boldsymbol{I}+\frac{\rho \boldsymbol{\rho}}{\rho^{2}}\right): \nabla_{\rho} \nabla_{\rho} \mathscr{P}_{1},
$$

where the non-homogeneous term on the right-hand side arises due to the $\nabla \cdot \boldsymbol{D}_{r}$ is $O\left(r^{-5}\right)$ and $\mathscr{P}_{1}$ is given by $O\left(r^{-1}\right)$ spatial dependence of $\boldsymbol{D}_{r}$ after noting that (A 38).

The solution of (A 39) is a fairly complicated task and, therefore, we adopt the numerical approach of Brady \& Vicic (1995) and compute the distortion of the microstructure using the spherical harmonic expansion (A 30), followed by the numerical integration according to (A 31). The results for $\phi^{-1} \Delta S_{0}$ vs. $P e$ are presented in figure 11 upon varying the truncation level $L$. The convergence of the results with respect to $L$ is slow, possibly due to slow decay of $\boldsymbol{D}_{r} \sim O\left(r^{-1}\right)$ in (A9), and already more than 30 harmonics are required in (A 30) for accurate computation of the microstructural deformation at $P e \sim 1$, when hydrodynamic interactions are taken into consideration. Therefore, the method of Bergenholtz, Brady \& Vicic (2002) based on a finite difference scheme, was found to be more useful for numerically evaluating the microstructural deformation in the long-wavelength limit for large $P e$. The corresponding results are depicted in the inset in figure 11. It is readily seen, that when hydrodynamic interactions are included, $\phi^{-1} \Delta S$ is positive up to $P e \approx 1.5$, in contrast to the case when hydrodynamic interactions are neglected (see figure 8). At large $P e$ the flow-induced microstructural deformation $\phi^{-1} \Delta S_{0}$ is negative and diminishes with the rate $\sim \log P e$ vs. the $\sqrt{P e}$-dependence found when hydrodynamics are neglected (see figure 9). The viscous forces act to narrow the wakes of depleted $g(\boldsymbol{r})$ and result in a more efficient angular diffusion. Thus, intuitively, smaller microstructural deformations are expected for hydrodynamically interacting particles than for the case without hydrodynamic interaction at high $\mathrm{Pe}$. 


\section{REFERENCES}

Acrivos, A., Batchelor, G. K., Hinch, E. J., Koch, D. L. \& Mauri, R. 1992 The longitudinal shear-induced diffusion of spheres in a dilute suspension. J. Fluid Mech. 240, 651-657.

BAtchelor, G. K. 1972 Sedimentation in a dilute dispersion of spheres. J. Fluid Mech. 52, 245-268.

BATCHELOR, G. K. 1976 Brownian diffusion of particles with hydrodynamic interaction. J. Fluid Mech. 74, 1-29.

BAtChelOR, G. K. 1977 The effect of Brownian motion on the bulk stress in a suspension of spherical particles. J. Fluid Mech. 83, 97-117.

BAtCheloR, G. K. 1979 Mass transfer from a particle suspended in fluid with a steady linear ambient velocity distribution. J. Fluid Mech. 95, 369-400.

Batchelor, G. K. \& Green, J. T. 1972 The hydrodynamic interactions of two small freely-moving spheres in a linear flow field. J. Fluid Mech. 56, 375-400.

Bergenholtz, J., Brady, J. F. \& Vicic, M. A. 2002 The non-Newtonian rheology of dilute colloidal suspensions. J. Fluid Mech. 456, 239-275.

Berne, B. J. \& Pecora, R. 1976 Dynamic Light Scattering. Wiley.

BEawzdziewicz, J. \& Szamel, G. 1993 Structure and rheology of semidilute suspensions under shear Phys. Rev. E. 48, 4632-4636.

Brady, J. F. 1994 The long-time self-diffusivity in concentrated colloidal dispersions. J. Fluid Mech. 272, 109-134.

Brady, J. F. \& Morris, J. F. 1997 Microstructure of strongly sheared suspensions and its impact on rheology and diffusion. J. Fluid Mech 348, 103-139.

Brady, J. F., Phillips, R. J., Lester, J. C. \& Bossis, G. 1988 Dynamic simulation of hydrodynamically interacting suspensions. J. Fluid Mech. 195, 257-280.

BRADY, J. F. \& Vicic, M. 1995 Normal stresses in colloidal dispersions. J. Rheol. 40, 545-566.

Breedveld, V., van den Ende, D., Triphati, A. \& Acrivos, A. 1998 The measurement of the shear-induced particle and fluid tracer diffusivities by a novel method. J. Fluid Mech. 375, 297-318.

CAflisch, R. E. \& LuKe, J. H. C. 1985 Variance in the sedimentation speed of a suspension. Phys. Fluids 28, 759-760.

DA CUnha, F. R. \& Hinch, E. J. 1996 Shear-induced disperion in a dilute suspension of rough spheres. J. Fluid Mech. 309, 211-223.

Dhont, J. K. G. 1996 An Introduction to the Dynamics of Colloids. Elsevier.

Drazer, G., Koplik, J., Khusid, B. \& Acrivos, A. 2002 Deterministic and stochastic behaviour of non-Brownian spheres in sheared suspensions. J. Fluid Mech. 460, 307-335.

Eckstein, E. C., Bailey, D. C. \& Shapiro, A. H. 1977 Self-diffusion of particles in shear-flow of a suspension. J. Fluid Mech. 79, 191-208.

EINSTEIN, A. 1905 On the movement of small particles suspended in stationary liquids required by the molecular-kinetic theory of heat. Annalen der Phys. 17, 549-560.

Elrick, D. E. 1962 Source functions for diffusion in uniform shear flow. Austral. J. Phys. 15, 283-288.

Foss, D. R. \& Brady, J. F. 1999 Self-diffusion in sheared suspensions by dynamic simulation. J. Fluid Mech. 401, 243-274.

Frankel, I. \& Brenner, H. 1991 Generalized Taylor dispersion phenomena in unbounded homogeneous shear flows. J. Fluid Mech. 230, 147-181.

Heyes, D. M. \& Melrose, J. R. 1993 Brownian dynamics simulations of model hard-sphere suspensions. J. Non-Newtonian Fluid Mech. 46, 1-28.

HINCH, E. J. 1977 An averaged-equation approach to particle interactions in a fluid suspension. J. Fluid Mech. 83, 695-720.

JEFFREY, D. J. \& ONISHI, Y. 1984 Calculation of the resistance and mobility functions for two unequal rigid spheres in low-Reynolds-number flow. J. Fluid Mech. 139, 261-290.

Johnson, S. J., DE Kruif, C. G. \& MAY, R. P. 1988 Structure factor distortion for hard-sphere dispersions subjected to weak shear flow: Small-angle neutron scattering in the flow-vorticity plane. J. Chem. Phys. 89, 5909-5921.

Kim, S. \& Karrila, S. J. 1991 Microhydrodynamics: Principles and Selected Applications. Butterworth-Heinemann. 
de Kruif, C. G., van der Werff, J. C., Johnson, S. J. \& May, R. P. 1990 Small-angle neutron scattering of sheared concentrated dispersions: Microstructure along principal axes. Phys. Fluids A 2, 1545-1556.

Leighton, D. T. \& ACRIVOS, A. 1987 The shear-induced migration of particles in concentrated suspensions. J. Fluid Mech. 181, 415-439.

Leshansky, A. M. \& Brady, J. F. 2005 Dynamic structure factor study of diffusion in strongly sheared suspensions. J. Fluid Mech. 527, 141-169.

Marchioro, M. \& ACrivos, A. 2001 Shear-induced particle diffusivities from numerical simuations. J. Fluid Mech. 443, 101-128.

MaURI, R. 2003 The constitutive relation of suspensions of non-colloidal particles in viscous fluids. Phys. Fluids 15, 1888-1896.

San Miguel, M. \& Sancho, J. M. 1979 Brownian motion in shear flow. Physica A 99, 357-364.

Morris, J. F. \& Brady, J. F. 1996 Self-diffusion in sheared suspensions. J. Fluid Mech. 312, 223-252.

Morris, J. F. \& KatYal, B. 2002 Microstructure from simulated Brownian suspension flows at large shear rate. Phys. Fluids 14, 1920-1937.

Novikov, E. A. 1958 Concerning turbulent diffusion in a stream with transverse gradient of velocity. Prikl. Mat. Mech. 22, 412-414 (in Russian).

Pusey, P. N. 1991 Colloidal suspensions. In Liquids, Freezing and Glass Transitions (ed. J. P. Hansen, D. Levesque \& J. Zinn-Justin). Elsevier.

Rallison, J. M. \& Hinch, E. J. 1986 The effect of particle interactions on dynamic light scattering from a dilute suspension. J. Fluid Mech. 167, 131-168.

Russel, W. B. \& Glendinning, A. B. 1981 The effective diffusion coefficient detected by dynamic light scattering. J. Chem. Phys. 72, 948-952.

Russel, W. B., Saville, D. A. \& Schowalter, W. R. 1989 Colloidal Dispersions. Cambridge University Press.

Schaertl, W. \& Sillescu, H. 1994 Brownian dynamics simulations of colloidal hard spheres. Effects of sample dimensionality on self-diffusion. J. Statist. Phys. 74, 687-703.

Segré, P. N., Behrend, O. P. \& Pusey, P. N. 1995 Short-time Brownian motion in colloidal suspenions: Experiment and simulation. Phys. Rev. E 52, 5070-5083.

Sierou, A. \& Brady, J. F. 2001 Accelerated Stokesian Dynamics simulations. J. Fluid Mech. 448, $115-146$.

Sierou, A. \& Brady, J. F. 2004 Shear-induced self-diffusion in non-colloidal suspensions. J. Fluid Mech. 506, 285-314.

TAYLOR, G. I. 1953 Dispersion of soluble matter in solvent flowing slowly through a tube. Proc. Roy. Soc. Lond. A 219, 186-203.

Vermant, J. \& Solomon, M. J. 2005 Flow induced structure in colloidal suspensions. J. Phys.-Cond. Matter 17, R187-R216.

VICIC, M. 1999 Rheology and microstructure of complex fluids: Dispersions, emulsions and polymer solutions. PhD dissertation, California Institute of Technology.

WAGNER, N. J. \& Russel, W. B. 1990 Light scattering experiments of a hard-sphere suspension under shear. Phys. Fluids A 2, 491-502.

WANG, Y. \& MAURI, R. 1999 Longitudinal shear-induced gradient diffusion in a dilute suspension of spheres. Intl J. Multiphase Flow 25, 875-885.

WANG, Y., MAURI, R. \& ACRIVOS, A. 1996 The transverse shear-induced liquid and particle tracer diffusivities of a dilute suspension of spheres undergoing a simple shear flow. J. Fluid Mech. 327, 255-272.

Wang, Y., Mauri, R. \& Acrivos, A. 1998 Transverse shear-induced gradient diffusion of a dilute suspension of spheres. J. Fluid Mech. 357, 279-287. 